\title{
An Adaptive Approach to Mitigate Background Covariance Limitations in the Ensemble Kalman Filter
}

\author{
HAJOON SONG \\ Scripps Institution of Oceanography, University of California, San Diego, La Jolla, California \\ IBRAHIM HOTEIT \\ King Abdullah University of Science and Technology, Thuwal, Saudi Arabia \\ Bruce D. Cornuelle AND ANEesh C. Subramanian \\ Scripps Institution of Oceanography, University of California, San Diego, La Jolla, California
}

(Manuscript received 18 November 2008, in final form 31 January 2010)

\begin{abstract}
A new approach is proposed to address the background covariance limitations arising from undersampled ensembles and unaccounted model errors in the ensemble Kalman filter (EnKF). The method enhances the representativeness of the EnKF ensemble by augmenting it with new members chosen adaptively to add missing information that prevents the EnKF from fully fitting the data to the ensemble. The vectors to be added are obtained by back projecting the residuals of the observation misfits from the EnKF analysis step onto the state space. The back projection is done using an optimal interpolation (OI) scheme based on an estimated covariance of the subspace missing from the ensemble. In the experiments reported here, the OI uses a preselected stationary background covariance matrix, as in the hybrid EnKF-three-dimensional variational data assimilation (3DVAR) approach, but the resulting correction is included as a new ensemble member instead of being added to all existing ensemble members.

The adaptive approach is tested with the Lorenz-96 model. The hybrid EnKF-3DVAR is used as a benchmark to evaluate the performance of the adaptive approach. Assimilation experiments suggest that the new adaptive scheme significantly improves the EnKF behavior when it suffers from small size ensembles and neglected model errors. It was further found to be competitive with the hybrid EnKF-3DVAR approach, depending on ensemble size and data coverage.
\end{abstract}

\section{Introduction}

Data assimilation (DA) combines numerical models and data to determine the best possible estimate of the state of a dynamical system (Ghil and Malanotte-Rizzoli 1991). The DA methods were historically grouped into two categories: variational methods based on optimal control theory, which seek the model trajectory that best fit the data over a given period of time (Le Dimet and Talagrand 1986); and sequential methods based on statistical estimation theory, which optimally combine model outputs and the most recent data according to their respective uncertainties (Todling 1999).

Corresponding author address: Hajoon Song, Scripps Institution of Oceanography, University of California, San Diego, 9500 Gilman Dr., La Jolla, CA 92093-0224.

E-mail: j0song@ucsd.edu
The Kalman filter (KF) is a sequential DA scheme that provides the optimal state estimate for linear models with Gaussian errors (Kalman 1960). The KF alternates a forecast step to integrate the most recent estimate forward in time with an analysis step to update (and correct) the forecast with new observations. However, the use of the $\mathrm{KF}$ for realistic atmospheric and oceanic problems is not feasible for two reasons (Ghil and Malanotte-Rizzoli 1991): the nonlinearity of these systems and the huge state dimensions (typically on the order of $10^{8}-10^{9}$ ). The first means that the KF is not optimal, and the second makes the computational burden for manipulating the error covariance matrices needed for the filter algorithm prohibitive.

The ensemble Kalman filter (EnKF) has been introduced by Evensen (1994) to solve the problems with the KF. It represents the uncertainties around the KF state 
estimates by an ensemble of state vectors, in place of computing the filter error covariance matrices. The time update of the uncertainties is carried out through the integration of the ensemble with the full nonlinear forward model, avoiding some of the problems that arise from the linearization of the system (Evensen 1994). The current uncertainty in the model parameter estimates is represented by the sample covariance matrix of the ensemble, also known as the background covariance. Accurate description of the background covariance matrix is critical for the performance of any data assimilation scheme as it describes the spatial and multivariate structure of the analysis increment (Lorenc 2003; Hamill and Snyder 2000).

Accounting for model deficiencies and small ensemble size remain a significant problem for EnKF assimilation, as they limit the accuracy of the estimated background covariance. Neglecting model errors results in underestimates of the background covariance matrix and ensemble spread, which degrades the fit to observations (Hamill and Whitaker 2005). Various techniques (e.g., covariance inflation and relaxation, and additive stochastic noise) have been used to partially compensate for the negative effects of model errors in the EnKF (Hamill and Whitaker 2005; Hoteit et al. 2007). These techniques require a priori knowledge about the nature and the statistics of all sources of uncertainties in the model, which is not often available. Despite continuous progress in computing capabilities, application of the EnKF to computationally demanding models, such as realistic atmospheric and oceanic models, still incurs large computational costs for integrating the ensemble forward in time. This sets severe limits on the size of the ensemble that can be used in practice. Running a small ensemble implies that the EnKF sample covariance matrices will have "low rank,"1 meaning that they only span a small region of the state space. Because the KF correction is only applied along the $N-1$ directions (where $N$ is the size of the ensemble) of the subspace generated by the ensemble (Pham 2001; Hoteit et al. 2002), the filter's correction may not be able to efficiently fit the observations. This problem, called the rank deficiency problem of the EnKF (Houtekamer and Mitchell 1998; Hamill and Snyder 2000), can impair the performance of the filter analysis in realistic applications where the number of members that can be integrated by the model is much smaller (typically on the order of 10-100 members) than the rank of the state error as reflected by the misfits with observations (can

\footnotetext{
${ }^{1}$ The rank of the sample covariance matrix is at most equal to the size of the ensemble - 1 (Pham 2001).
}

be of the order of thousands). Localization of the filter correction was first used in the context of the EnKF by Houtekamer and Mitchell (1998) as a way to "artificially" increase the effective rank of the covariance matrix without using more ensemble members. Localization should be used carefully because it might introduce undesirable artifacts into the analysis (Hamill and Snyder 2000) and eliminate real nonlocal correlations created by the sampling and the dynamics (Hoteit et al. 2001).

Neglecting model errors and the use of small ensembles generally mean that a significant part of state space is not represented by the ensemble, producing unrealistic confidence in the filter forecast. In both cases, important features may be omitted from the EnKF analysis, which might degrade the filter behavior or even lead to divergence in certain situations. The hybrid EnKFthree-dimensional variational data assimilation (3DVAR) or EnKF-optimal interpolation (OI) approach was introduced by Hamill and Snyder (2000) to reduce the impact of ensemble sampling errors in the EnKF. This method adds an empirical, stationary covariance matrix $\mathbf{B}$ to the EnKF "flow dependent" sample covariance matrix (see Wang et al. 2007 for a review). The matrix B is assumed to represent the gravest modes of the system, ensuring that they are represented in the EnKF correction subspace. Although augmenting the ensemble by the modes of $\mathbf{B}$ in an ad hoc manner is not optimal, it at least guarantees that the EnKF correction is not discarding important features of the system. Adding B to the background covariance ideally also accounts for model errors. Hence, the choice of $\mathbf{B}$ is crucial and different forms have been tested in practice (Hamill and Snyder 2000; Lorenc 2003; Buehner 2005).

In this work, we introduce a new, but closely related, adaptive approach to improve the representativeness of the EnKF ensemble by "enriching" it with members estimated from the missing part of the state space that prevents the EnKF from fitting the data. Adaptivity has been already used by Mitchell et al. (2002), but in the context of the EnKF to estimate parameters controlling the model error covariance matrix while tuning the innovation covariance matrix. The vectors that will be added to the ensemble are obtained by back projecting the residuals of the analysis step from data space to state space. The transformation to state space is achieved using an OI scheme based on preselected stationary background covariance matrix B, as in the hybrid EnKF3DVAR approach. In contrast with the hybrid approach, the new approach targets specific directions of $\mathbf{B}$ to enrich the EnKF ensemble. This should minimize the unnecessary structure in the analysis by limiting the augmentation of the background covariance matrix $\mathbf{B}$. 
The EnKF and OI analysis steps are further applied separately, which offers more numerical and implementation flexibility. For instance, a 3DVAR step can be used instead of an OI for complex forms of $\mathbf{B}$.

The paper is organized as follows. After briefly recalling the characteristics of the EnKF, the new adaptive EnKF is described in section 2. Section 3 presents a theoretical derivative and justification of the adaptive approach and compares it to the hybrid EnKF-3DVAR. Results of numerical experiments with the Lorenz-96 model (Lorenz and Emanuel 1998) are then presented and discussed in section 4 , followed by a general discussion to conclude in section 5 .

\section{The adaptive ensemble Kalman filter}

Starting from an initial set of ensemble states that supposedly represents the uncertainties about the initial state estimate, the EnKF operates as a succession of two steps: a forecast step to integrate the ensemble with the model forward in time, and an analysis step to update each member of the forecast ensemble with the new observations. After an analysis or a forecast step, the mean of the ensemble is the filter estimate. The EnKF analysis is linear and is based on that of the KF. The analysis ensemble can be written in matrix form as in Evensen (2003):

$$
\mathbf{X}^{a}=\mathbf{X}^{f}+\mathbf{K}\left(\mathbf{D}-\mathbf{H} \mathbf{X}^{f}\right),
$$

where $\mathbf{X}^{a}$ and $\mathbf{X}^{f}$ are the matrices whose columns are the analysis and forecast ensembles, respectively. The $\mathbf{D}$ is the matrix whose columns are the observation vector d perturbed with independent random noise generated from the probability distribution function of the observational errors. The perturbed observations are introduced so that the EnKF analysis exactly matches the KF analysis (Burgers et al. 1998). The $\mathbf{H}$ is the linearized observational operator relating the model state to the observations. The $\mathbf{K}$ is the weighting matrix, also called the Kalman gain. It provides the best analysis among linear estimates and is given by

$$
\mathbf{K}=\mathbf{P H}^{\mathrm{T}}\left(\mathbf{H} \mathbf{P H}{ }^{\mathrm{T}}+\mathbf{R}\right)^{-1},
$$

where $\mathbf{R}$ is the observational error covariance matrix, and $\mathbf{P}$ is the sample covariance matrix of the forecast ensemble. For an ensemble of $N$ members,

$$
\mathbf{P}=\frac{1}{N-1}\left(\mathbf{X}^{f}-\overline{\mathbf{X}}^{f}\right)\left(\mathbf{X}^{f}-\overline{\mathbf{X}}^{f}\right)^{\mathrm{T}}
$$

with $\overline{\mathbf{X}}^{f}$ is the matrix whose columns are the mean of the forecast ensemble. Explicit computation of $\mathbf{P}$ is not needed if one computes $\mathbf{P} \mathbf{H}^{\mathrm{T}}$ and $\mathbf{H} \mathbf{P} \mathbf{H}^{\mathrm{T}}$ with

$$
\begin{aligned}
\mathbf{P H}^{\mathrm{T}} & =\frac{1}{N-1}\left(\mathbf{X}^{f}-\overline{\mathbf{X}}^{f}\right)\left[\mathbf{H}\left(\mathbf{X}^{f}-\overline{\mathbf{X}}^{f}\right)\right]^{\mathrm{T}}, \\
\mathbf{H P H} & =\frac{1}{N-1}\left[\mathbf{H}\left(\mathbf{X}^{f}-\overline{\mathbf{X}}^{f}\right)\right]\left[\mathbf{H}\left(\mathbf{X}^{f}-\overline{\mathbf{X}}^{f}\right)\right]^{\mathrm{T}},
\end{aligned}
$$

as suggested by Houtekamer and Mitchell (1998). When the model is not perfect, $\mathbf{P}$ is augmented by the covariance matrix of the model uncertainties.

Pham (2001) argued that if large enough ensembles are used, ensemble Kalman filters should be stable with linear perfect autonomous systems. This is because the filter correction is made along the directions of the subspace generated by the ensemble, which presumably evolve toward the fastest-growing modes of the system. In this case, one has only to use more ensemble members than the number of growing modes of the system. In realistic atmospheric and oceanic applications, updating the ensemble forward in time has large computational costs due to the huge dimensions of these systems. The cost limits the size of the ensemble that can be considered in practice (Houtekamer and Mitchell 1998; Hoteit et al. 2002). Using small ensembles raises the risk of omitting the filter correction for some important modes, which might degrade the filter's behavior. Hence the EnKF is characterized by its "rank deficient" covariance matrices (Hamill and Snyder 2000). Moreover, most dynamical models encountered in practice, as in meteorology and oceanography, are not autonomous and are imperfect. The number and the directions of the growing modes might therefore vary in time, especially during some unstable periods (Hoteit and Pham 2004). In this case, it is difficult for the EnKF ensemble to follow newly triggered modes, and the filter behavior might be seriously degraded during these periods. In the following, we introduce a new adaptive approach to mitigate the background covariance limitations in the EnKF.

To describe the adaptive approach, we first define the residual vector $\mathbf{r}$ as the difference between the filter analysis $\mathbf{x}^{a}$ (the mean of the analysis ensemble $\mathbf{X}^{a}$ ) projected into the observation space and the observations:

$$
\mathbf{r}=\mathbf{d}-\mathbf{H} \mathbf{x}^{a} .
$$

In addition to observational errors, the residuals are the result of the missing directions in the ensemble and poorly known model errors. The residuals carry information about the part of the state space that prevents the filter estimate from fully fitting the observations. The goal of the adaptive method is to enrich the ensemble with this information in order to enhance its representativeness. Anderson (1996b) suggested that the projection of the estimation error on the attractor of the Lorenz model is the most effective selection of initial conditions 
for ensemble forecast. In the same manner, the residuals need to be transformed from the observational space to the state space. An efficient way to do that is to solve the following three-dimensional assimilation problem where we seek the vector $\delta \mathbf{x}^{e}$ that optimizes the cost function:

$$
J\left(\delta \mathbf{x}^{e}\right)=\frac{1}{2}\left(\delta \mathbf{x}^{e}\right)^{\mathrm{T}} \mathbf{B}^{-1} \delta \mathbf{x}^{e}+\frac{1}{2}\left(\mathbf{r}-\mathbf{H} \delta \mathbf{x}^{e}\right)^{\mathrm{T}} \mathbf{R}^{-1}\left(\mathbf{r}-\mathbf{H} \delta \mathbf{x}^{e}\right),
$$

with $\mathbf{B}$ a covariance matrix representing the uncertainty that is missing from the ensemble. This matrix controls the projection of the residuals from the observation space to the state space. The use of the residuals to select the ensemble members to be added is meant to limit the growth of the ensemble to directions indicated by the observations but missing from the ensemble. The optimal solution of this problem is given by (Lorenc 1986)

$$
\delta \mathbf{x}^{e}=\mathbf{B H}^{\mathrm{T}}\left(\mathbf{H B H} \mathbf{H}^{\mathrm{T}}+\mathbf{R}\right)^{-1} \mathbf{r} .
$$

The posterior covariance is then

$$
\mathbf{B}^{a}=\mathbf{B}-\mathbf{B H}^{\mathrm{T}}\left(\mathbf{H B H}^{\mathrm{T}}+\mathbf{R}\right)^{-1} \mathbf{H B} .
$$

An optimization algorithm can be used to optimize $J$ in (7) if the matrix $\left(\mathbf{H}^{\mathrm{T}} \mathbf{B H}+\mathbf{R}\right)$ is not easily invertible. Once $\delta \mathbf{x}$ is computed, the analysis ensemble $\mathbf{X}^{a}$ is augmented by the new member:

$$
\mathbf{x}^{a, e}=\mathbf{x}^{a}+\beta \delta \mathbf{x}^{e} .
$$

The tuning factor $\beta$ is included as a way to set the weight of the new member in the ensemble. Larger values of $\beta$ shift the mean of the new ensemble toward a state that fits the observations. Setting $\beta=N$, the (new) number of ensemble elements, means that the average of the new ensemble has been shifted by $\delta \mathbf{x}^{e}$. In experiments, however, $\beta=N$ often diverged, and $\beta$ in the range from 1 to 2 was found to work well with small ensembles, although this may depend on the particular choice of system and observations. A more thorough discussion on the choice of the parameter $\beta$ is given in section $4 \mathrm{c}$.

In the case of a perfect ensemble, the residuals with a perfect $\mathbf{R}$ are expected to be mainly due to observational errors after some assimilation cycles. If the residuals are known to be nearly pure observational errors, then $\mathbf{B}$ should be small. That means $\mathbf{H B H}^{\mathrm{T}} \ll \mathbf{R}$, and the transformation of the residuals to the state space by the optimal interpolation step including $\mathbf{R}$ should produce a small $\delta \mathbf{x}^{e}$. Hence, there would be no point in doing the adaptive step if the distribution of the residuals was consistent with $\mathbf{R}$. If $\mathbf{B}$ is mistakenly taken to be large when it should be small, then the estimate will not be small and noise will become part of the ensemble. Noise is already in the EnKF to represent observational errors and this new method seems no more vulnerable to mis-specified priors than others.

We are interested in the cases where the ensemble is not perfect, so that the evidence for missing elements can be found in the residuals. In this study we included random errors in the observations, so the effectiveness of our procedure in the presence of observational errors was implicitly tested. Since our approach differs from the "classic" EnKF only in the method for ensemble updating, its sensitivity to observational errors and misspecification of $\mathbf{R}$ should be similar to the EnKF.

The correction $\delta \mathbf{x}^{e}$ is optimal [in the sense of (7)] given the residuals, the sampling, the observational error covariance, and the background covariance. When observations are sparse, the single estimate may not resolve the correct direction in state space. Alternatively, a set of new ensemble elements can be taken from the first few conjugate gradient descent directions in an iteration minimizing $J$. This would give more weight on the steepest-descent direction and would allow the addition of more than one ensemble element, reducing the effects of the weighting matrix B. Another approach would be to (randomly) sample more new members from the a posteriori distribution of the estimated $\delta \mathbf{x}^{e}$. One way to sample $m$ new members is to first apply a singular value decomposition to compute a low $m-1$ rank approximation of the posterior covariance matrix $\mathbf{B}^{a}$ of $\delta \mathbf{x}^{e}$, and then to use a second-order exact sampling scheme that preserves the first two moments of the (approximate) distribution to draw the $m$ members as described by Pham (2001). The later approach was used in the experiments discussed in section $4 \mathrm{c}$.

Adding even one member to the ensemble after every analysis step would quickly grow the computational burden of integrating the new ensemble to a prohibitive level. To avoid growth, members should be dropped from the ensemble to limit its size. One heuristic approach is to drop the member(s) that contribute the least to the ensemble spread, and so presumably carry the least amount of information. In the present study, the closest member to the ensemble mean was removed after every analysis step. Distances between the ensemble mean and the members were determined by the Euclidean norm normalized by the standard deviations of the model variables from a long run.

\section{Mathematical basis and relation to existing methods}

This section presents a mathematical basis for the adaptive approach and discusses its theoretical relation 
with the hybrid and inflation-localization approaches. The derivation includes an ad hoc choice between what to add to existing ensemble elements and what to convert to a new element.

Because the ensemble is only an approximation to the true uncertainties around the state estimate, the true error covariance $\mathbf{P}^{t}$ can be written formally as

$$
\mathbf{P}^{t}=\mathbf{P}+\mathbf{P}^{r},
$$

where $\mathbf{P}$ is the ensemble covariance given by (3) and $\mathbf{P}^{r}$ is the error in the ensemble estimate of the true covariance. Usually the ensemble is thought to cover only a subspace of the true covariance, so the error is a nonnegative definite covariance matrix for the remaining subspace, but this is not required in the following derivation.

Hybrid, covariance inflation, and covariance localization methods are all used to estimate $\mathbf{P}^{r}$, and any or all could be used to approximately account for the underestimation and the rank deficiency of the EnKF. The hybrid method of Hamill and Snyder (2000) relaxes the flow-dependent covariance matrix of the EnKF to B using a weighting parameter $\alpha$, so that the estimated true error covariance $\tilde{\mathbf{P}}^{t}$ is

$$
\tilde{\mathbf{P}}^{t}=\alpha \mathbf{P}+(1-\alpha) \mathbf{B},
$$

as a way to represent the subspace missing from the ensemble. The weighting parameter $\alpha$ takes values between 0 and 1.

Covariance inflation algorithms increase the ensemble variance by pushing ensemble members away from the ensemble mean (Anderson and Anderson 1999). Localization algorithms reduce the impact of distant observation on a state variable (Houtekamer and Mitchell 1998). This reduces spurious correlations and increases the rank of the localized covariance matrix, allowing improved fits to observations. These techniques were used in the assimilation experiments presented in section 4. The $\mathbf{P}^{r}$ matrix is not explicit in localization, but could be obtained in principle by subtracting the original covariance matrix from the localized matrix. This may not be practical and is not necessary.

Using the true covariance, the ideal Kalman gain would be

$$
\mathbf{K}^{t}=\mathbf{P}^{t} \mathbf{H}^{\mathrm{T}}\left(\mathbf{H P}^{t} \mathbf{H}^{\mathrm{T}}+\mathbf{R}\right)^{-1},
$$

or, splitting $\mathbf{P}^{t}$ into ensemble and "missing" subspaces,

$$
\mathbf{K}^{t}=\left(\mathbf{P}+\mathbf{P}^{r}\right) \mathbf{H}^{\mathrm{T}}\left(\mathbf{H} \mathbf{P H} \mathbf{H}^{\mathrm{T}}+\mathbf{H P}^{r} \mathbf{H}^{\mathrm{T}}+\mathbf{R}\right)^{-1} .
$$

Note that the gain for the estimate of the residuals that is not part of the ensemble is

$$
\mathbf{K}^{R}=\mathbf{R}\left(\mathbf{H P H} \mathbf{H}^{\mathrm{T}}+\mathbf{H P}^{r} \mathbf{H}^{\mathrm{T}}+\mathbf{R}\right)^{-1}
$$

where the subspace missing from the ensemble acts as an extra "noise" term inside the inverse, $\mathbf{R}^{e}=\mathbf{H P}^{r} \mathbf{H}^{\mathrm{T}}$.

Localization, inflation, and hybrid methods all use the estimated $\mathbf{K}^{t}$ as the gain for the ensemble elements, although the estimated $\mathbf{P}^{t}$, which includes $\mathbf{P}^{r}$, is no longer the covariance of the ensemble elements in these methods. In contrast, the adaptive approach separates the analysis step in (1) into a step updating the ensemble and one estimating a new element. To see that, one can use the ensemble covariance $\mathbf{P}$ in the analysis step so that

$$
\mathbf{K}=\mathbf{P} \mathbf{H}^{\mathrm{T}}\left(\mathbf{H} \mathbf{P} \mathbf{H}^{\mathrm{T}}+\mathbf{H P}^{r} \mathbf{H}^{\mathrm{T}}+\mathbf{R}\right)^{-1}
$$

and estimate an extra vector $\delta \mathbf{x}^{e}$ using $\mathbf{P}^{r}$ in the analysis gain:

$$
\mathbf{K}^{r}=\mathbf{P}^{r} \mathbf{H}^{\mathrm{T}}\left(\mathbf{H} \mathbf{P} \mathbf{H}^{\mathrm{T}}+\mathbf{H P}^{r} \mathbf{H}^{\mathrm{T}}+\mathbf{R}\right)^{-1}
$$

Thus,

$$
\delta \mathbf{x}^{e}=\mathbf{K}^{r}\left(\mathbf{d}-\mathbf{H}^{f}\right) .
$$

This $\mathbf{K}^{r}$ is like the residual gain, so the estimate $\delta \mathbf{x}^{e}$ can be thought of the model space transformation of the residuals caused by an inadequate ensemble. Viewed this way, it should not be added to the existing ensemble elements.

If $\mathbf{P}^{r}$ is entirely used in the ensemble update step, as in current methods, the correction increments are added to all ensemble members. The proposed approach allows a choice between adding similar increments to all members and adding a new member [or replacing an old member with a new member-the adaptive ensemble Kalman filter (AEnKF) approach]. Although no rigorous general case for the superiority of the latter has been found, one can argue that in some applications the ensemble elements tend to converge to similar vectors in state space so that there is large correlation between members (Houtekamer and Mitchell 1998). If this is the case, then adding the similar increments to each member does not increase the rank of the ensemble, and adding a new member may be preferable. Of course, the random perturbations added to the observations (to make D) increase the rank of the ensemble, but these represent observational uncertainty.

In the AEnKF, the new ensemble is then written as a concatenation of the old ensemble in (1) and the new element is

$$
\mathbf{X}^{a, e}=\left[\mathbf{X}^{f}+\mathbf{K}\left(\mathbf{D}-\mathbf{H} \mathbf{X}^{f}\right) \vdots \mathbf{x}^{a}+\beta \delta \mathbf{x}^{e}\right] .
$$


Then, $\mathbf{X}^{a, e}$ is integrated to the next (forecast) time step through the fully nonlinear model just as in the EnKF. If necessary, the ensemble could be reduced in size before the next forecast step. Altering the ensemble so that the ensemble mean matches (17)-(18) would require setting $\beta=N$ in (10). This would, however, put too much weight on the new direction, especially when the ensemble size $N$ is large, and may further cause overspreading of the ensemble and dynamical imbalances. Using $\beta$ as a tuning parameter enhanced the filter behavior in our experiments, which supports the idea of improving the ensemble with a well-chosen direction as in the AEnKF.

It might be desirable to combine the adaptive approach with existing methods as localization and inflation. To do that, one can consider the previous partition in (11)-(17) to be a tunable parameter, where $\mathbf{P}^{r}$ is separated into two parts, $\mathbf{P}^{r}=\mathbf{P}^{\mathrm{r} 1}+\mathbf{P}^{\mathrm{r} 2}$, the first of which is used to update the ensemble, and the second of which is used to estimate the new element. The two analysis gains are then

$$
\mathbf{K}=\left(\mathbf{P}+\mathbf{P}^{\mathrm{r} 1}\right) \mathbf{H}^{\mathrm{T}}\left(\mathbf{H} \mathbf{P} \mathbf{H}^{\mathrm{T}}+\mathbf{H} \mathbf{P}^{r} \mathbf{H}^{\mathrm{T}}+\mathbf{R}\right)^{-1}
$$

for the ensemble and

$$
\mathbf{K}^{r}=\mathbf{P}^{\mathrm{r} 2} \mathbf{H}^{\mathrm{T}}\left(\mathbf{H} \mathbf{P} \mathbf{H}^{\mathrm{T}}+\mathbf{H P}^{r} \mathbf{H}^{\mathrm{T}}+\mathbf{R}\right)^{-1}
$$

for the new element.

The optimal choice for $\mathbf{P}^{\mathrm{r} 1}$ and $\mathbf{P}^{\mathrm{r} 2}$ has not been shown in this study. Trials could be run with $\mathbf{P}^{\mathrm{r} 1}=0$ and a $\mathbf{P}^{\mathrm{r} 2}$ constructed by both localization and an added $\mathbf{B}$, or both $\mathbf{P}^{\mathbf{r} 1}$ and $\mathbf{P}^{\mathbf{r} 2}$ could be weighted combinations of hybrid, localization, and inflation methods. In the experiments reported next, inflation, localization, and hybrid methods are used to make $\mathbf{P}^{\mathrm{r} 1}$. Hence, if $\mathbf{P}^{\mathbf{r}}=0$, then the hybrid, localized, or inflated EnKF is recovered, and no extra element is generated.

The $\delta \mathbf{x}^{e}$ in (8) is estimated from the observation misfit after the ensemble analysis. To put the estimate derived in (18) into the form of (8), we first substitute (21) into (18):

$$
\begin{aligned}
\delta \mathbf{x}^{e}= & \mathbf{P}^{\mathrm{r} 2} \mathbf{H}^{\mathrm{T}}\left(\mathbf{H} \mathbf{P} \mathbf{H}^{\mathrm{T}}+\mathbf{H P}^{r} \mathbf{H}^{\mathrm{T}}+\mathbf{R}\right)^{-1}\left(\mathbf{d}-\mathbf{H} \mathbf{x}^{f}\right) \\
= & \mathbf{P}^{\mathrm{r} 2} \mathbf{H}^{\mathrm{T}}\left(\mathbf{H P}^{\mathrm{r} 2} \mathbf{H}^{\mathrm{T}}+\mathbf{R}\right)^{-1}\left(\mathbf{H} \mathbf{P}^{\mathrm{r} 2} \mathbf{H}^{\mathrm{T}}+\mathbf{R}\right) \\
& \times\left(\mathbf{H} \mathbf{P} \mathbf{H}^{\mathrm{T}}+\mathbf{H P}^{r} \mathbf{H}^{\mathrm{T}}+\mathbf{R}\right)^{-1}\left(\mathbf{d}-\mathbf{H} \mathbf{x}^{f}\right) .
\end{aligned}
$$

Recalling that $\mathbf{P}^{\mathrm{r} 2}=\mathbf{P}^{r}-\mathbf{P}^{\mathrm{r} 1}$ and adding and subtracting the term $\mathbf{H P H}^{\mathrm{T}}$, the above equation can be rewritten as

$$
\begin{aligned}
\delta \mathbf{x}^{e} & =\mathbf{P}^{\mathrm{r} 2} \mathbf{H}^{\mathrm{T}}\left(\mathbf{H} \mathbf{P}^{\mathrm{r} 2} \mathbf{H}^{\mathrm{T}}+\mathbf{R}\right)^{-1}\left[\mathbf{I}-\mathbf{H}\left(\mathbf{P}+\mathbf{P}^{\mathrm{r} 1}\right) \mathbf{H}^{\mathrm{T}}\left(\mathbf{H P} \mathbf{H}^{\mathrm{T}}+\mathbf{H P}^{r} \mathbf{H}^{\mathrm{T}}+\mathbf{R}\right)^{-1}\right]\left(\mathbf{d}-\mathbf{H} \mathbf{x}^{f}\right) \\
& =\mathbf{P}^{\mathrm{r} 2} \mathbf{H}^{\mathrm{T}}\left(\mathbf{H} \mathbf{P}^{\mathrm{r} 2} \mathbf{H}^{\mathrm{T}}+\mathbf{R}\right)^{-1}\left\{\mathbf{d}-\mathbf{H}\left[\mathbf{x}^{f}+\left(\mathbf{P}+\mathbf{P}^{\mathrm{r} 1}\right) \mathbf{H}^{\mathrm{T}}\left(\mathbf{H} \mathbf{P} \mathbf{H}^{\mathrm{T}}+\mathbf{H} \mathbf{P}^{r} \mathbf{H}^{\mathrm{T}}+\mathbf{R}\right)^{-1}\left(\mathbf{d}-\mathbf{H} \mathbf{x}^{f}\right)\right]\right\} \\
& =\mathbf{P}^{\mathrm{r} 2} \mathbf{H}^{\mathrm{T}}\left(\mathbf{H} \mathbf{P}^{\mathrm{r} 2} \mathbf{H}^{\mathrm{T}}+\mathbf{R}\right)^{-1}\left\{\mathbf{d}-\mathbf{H}\left[\mathbf{x}^{f}+\mathbf{K}\left(\mathbf{d}-\mathbf{H} \mathbf{x}^{f}\right)\right]\right\}=\mathbf{P}^{\mathrm{r} 2} \mathbf{H}^{\mathrm{T}}\left(\mathbf{H} \mathbf{P}^{\mathrm{r} 2} \mathbf{H}^{\mathrm{T}}+\mathbf{R}\right)^{-1}\left(\mathbf{d}-\mathbf{H} \mathbf{x}^{a}\right) .
\end{aligned}
$$

This is exactly the same form as (8) with $\mathbf{P}^{\mathrm{r} 2}$ taking the place of $\mathbf{B}$. To exactly match the EnKF update in (1)-(2), the term $\mathbf{R}^{e}=\mathbf{H P}^{r} \mathbf{H}^{\mathrm{T}}$ must be omitted from the matrix inverted in the expression of the gain matrix in (16) used to update the ensemble, as in some approximate methods (Hamill et al. 2001).

The new vector estimated in (10) using (22) contains information missing from the ensemble, and including it as a new ensemble member in the EnKF is expected to enrich the ensemble with missing information, which is the main idea behind the AEnKF.

Localization, inflation, and hybrid (LIH) methods add freedom to fit the data by increasing the rank of the estimator and/or by amplifying the background error covariance, altering the gain $\mathbf{K}$, but do not increase the diversity of the ensemble directly in the EnKF since the increments added to the ensemble elements are similar except for the perturbed observations. In addition, all three methods ( $\mathrm{LIH})$ dilute the information contained in the ensemble by altering or augmenting the covariance. The ensemble defines the subspace in which the solution is thought to lie, and this information is expensively propagated between analysis steps. As the ensemble covariance is modified by LIH, this information is partially ignored, and the solution may be taken from a "broader" subspace with increased rank and/or variance.

The increased freedom to fit the observations comes at the expense of losing the information accumulated from previous observations. So, for example, the limiting case of using strong localization to reduce the residuals in the analysis step would create a diagonal data-data covariance matrix to be inverted. This would allow an excellent fit to the observations, but most information in the ensemble would be lost. The hypothesis motivating the AEnKF is that in some cases a subspace outside that spanned by the ensemble may grow, (e.g., because of a forcing error), and this will manifest itself as increasing residuals. The residuals can be decreased by LIH methods, but at the expense of some of the information in the ensemble. Back projecting the residuals to a new element preserves the information in the 
ensemble and increases diversity because the new vector is a back projection of a vector (the residuals), which is not contained in the forward projection of the ensemble subspace to observation space: $\mathbf{H X} \mathbf{X}^{f}$. AEnKF strives to balance preserving the covariance structure and diversifying the ensemble.

In the example shown below, the new elements are estimated using the hybrid method idea of representing the information missing from the sampled covariance matrix of the EnKF by an invariant 3DVAR background covariance. The adaptive approach does not use the "improved" background covariance matrix to correct the forecast ensemble as in the hybrid approach. Instead, it "complements" the EnKF error covariance by the 3DVAR background covariance. The projection of the analysis residual onto the subspace spanned by this matrix using the adjoint of the observational operator is then included as a new member in the ensemble. This new information is next propagated in time through the evolution of the ensemble by the nonlinear dynamics of the model. The parameter $\beta$ introduced in (10) plays a similar role to $\alpha$ in the hybrid approach in (12) in adjusting variance scaling.

\section{Numerical experiments}

\section{a. Model description and cases examined}

To study the behavior of the AEnKF, assimilation experiments were performed with the strongly nonlinear Lorenz-96 (L96) model (Lorenz and Emanuel 1998). This model is widely used in the assimilation community to test EnKF-based schemes (Whitaker and Hamill 2002) and provides a repeatable performance benchmark. Similar to the atmospheric system, L96 has energy conservation, nonlinear advection and linear dissipation, sensitivity to initial conditions, and external forcing. It is described by the following set of differential equations:

$$
\frac{d x(j, t)}{d t}=x(j-1, t)[x(j+1, t)-x(j-2, t)]-x(j, t)+F,
$$

where $j=1,2, \ldots, L$ and $x(-1, t)=x(L-1, t), x(0, t)=$ $x(L, t)$, and $x(L+1, t)=x(1, t)$, with the choice of $L=40$ and $F=8$. L96 was discretized here using Runge-Kutta fourth-order scheme with a time step $t=0.05$, which corresponds to $6 \mathrm{~h}$ in real time. For $F=8$, disturbances propagate from low to high indices ("west" to "east") and the model behaves chaotically (Lorenz and Emanuel 1998).

Our goal in using the L96 is to test the AEnKF while exploring the impact of ensemble size, model error, and observational coverage, and comparing its performance to the traditional EnKF and the hybrid EnKF-3DVAR. Because the example used to test the adaptive approach has similarities to the hybrid approach in many aspects, the performance of the hybrid EnKF-3DVAR was used as a reference to evaluate the performance of the AEnKF. As discussed in section 3, all three filters were implemented and tested with covariance inflation and covariance localization as described in Whitaker and Hamill (2002). Localization was implemented using the fifth-order function of Gaspari and Cohn (1999). This function behaves like a Gaussian function, but reaches zero at finite radius (Hamill et al. 2001). The setup of the assimilation experiments presented below is very similar to that of Whitaker and Hamill (2002), but we assimilated observations less frequently (every fourth time step, which is equivalent to 1 day in real time) to mimic more realistic situations. Three different observation strategies were also tested: observations sampled from all model variables, every other model variable, and every fourth model variable. For completeness, we made sure that we were able to reproduce the results of Whitaker and Hamill (2002) in our implementation of the filters. Observations were extracted from a reference model run and were perturbed with normally distributed noise of mean 0 and variance 1 . Accordingly, the observational error covariance $\mathbf{R}$ is set to the identity matrix.

To generate the filter initial conditions and the transformation matrix $\mathbf{B}$, the model was integrated for a long period of time, equivalent to several years in real time, without assimilation. The mean and the covariance from the run were used for the initial ensemble mean and the matrix B, respectively. Ideally, B should represent the uncertainty that is missing in the ensemble as stated after (7). In this experiment, we instead chose $\mathbf{B}$, which carries the uncertainty of the system, because it is more practical and is similar to the hybrid method. The starting ensemble members were generated by adding independent Gaussian random perturbations with unit variance to each model variable. The background error covariance for the EnKF and AEnKF was estimated according to (3), and according to (12) for the hybrid EnKF-3DVAR. The parameter $\alpha$ in (12) was set to 0.1 , after performing several assimilation runs with different values of $\alpha$, and the parameter $\beta$ in (10) was set to 1 unless it is specified. All assimilation experiments were integrated for 120 days (480 model steps), but only the last 100 days were considered in the analysis of the results to exclude an early spinup period of 20 days, similar to Whitaker and Hamill (2002).

Three different cases are separately discussed hereafter to better assess the behavior of the AEnKF when 
(a) EnKF, all

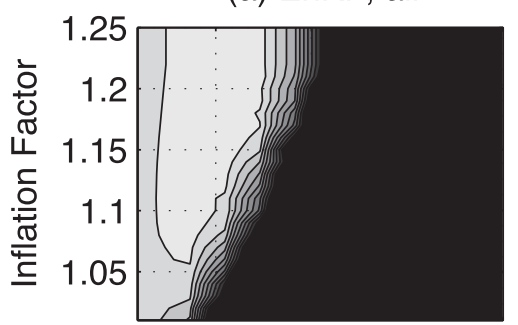

(d) Hybrid, all

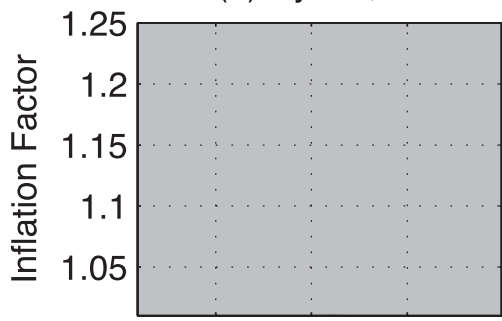

(g) AEnKF, all
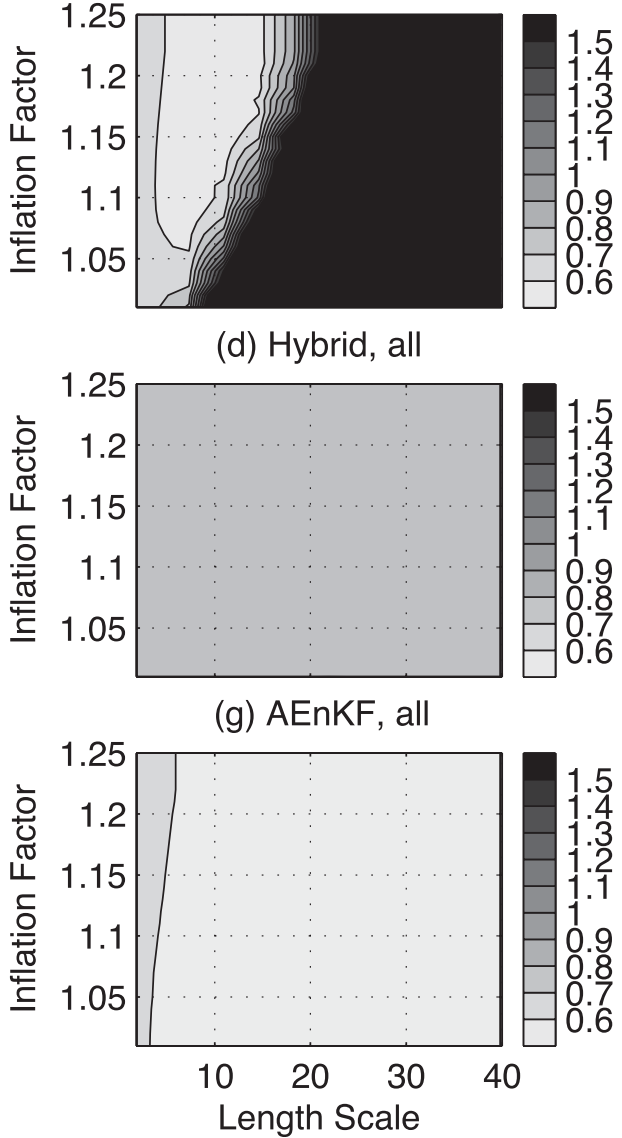

(b) EnKF, half
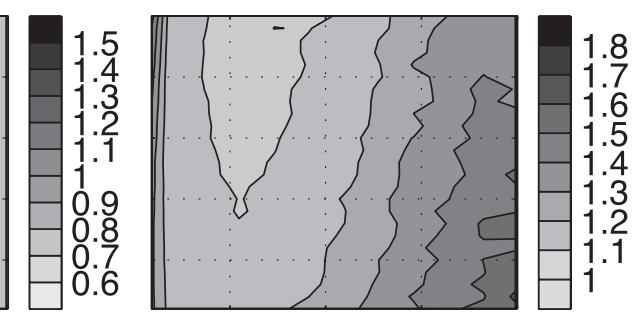

(h) AEnKF, half

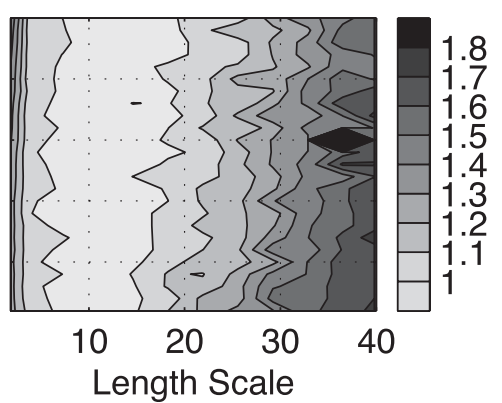

(c) EnKF, quarter

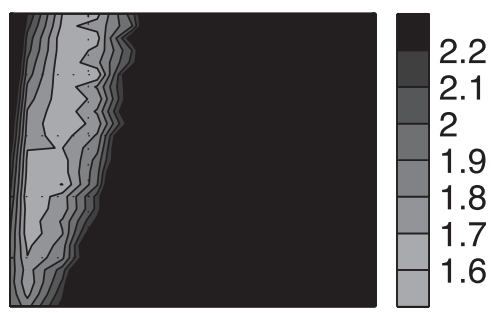

(f) Hybrid, quarter

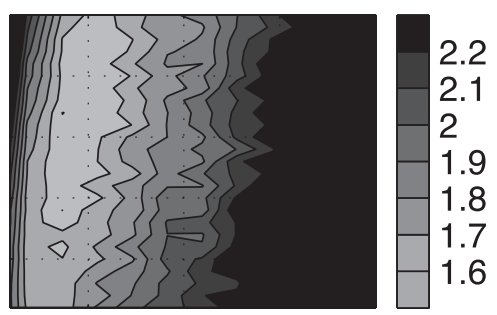

(i) AEnKF, quarter

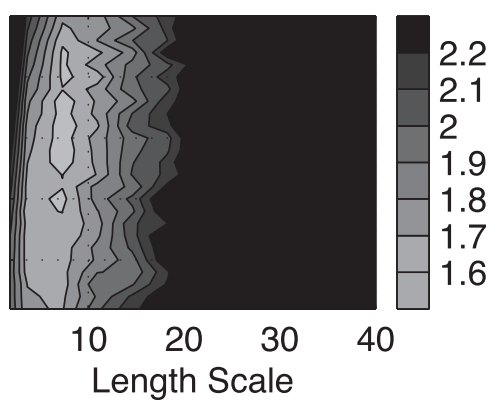

FIG. 1. RMSE-averaged over time and all variables as a function of length scale of the error covariance correlation and inflation factor. (top) EnKF, (middle) hybrid EnKF-3DVAR, and (bottom) AEnKF are implemented with 10 members and observations from (left to right) three different network densities were assimilated: all variables, every second variable, and every fourth variable at every fourth model time step (or 1 day in real time). No model error is introduced in these experiments.

the EnKF background covariance matrix suffers from errors due to sampling error only (or small ensemble), model error only (or large ensemble), and both sampling and model errors.

\section{b. Case with only sampling error}

In the first series of experiments, we examined the AEnKF in the situation where the accuracy of the EnKF covariance matrix only suffers from the sampling error (or small ensemble). The same model in (23) was used for the reference run and the filter forecast for the no model error case. The filters were implemented with a small ensemble of 10 members to introduce sampling error in their background covariance matrices.

Figure 1 shows the root mean-square error (RMSE) between the reference states and the filter estimates averaged over all 40 model variables and over the assimilation period as they result from the EnKF, AEnKF, and hybrid EnKF-3DVAR. The RMSE was plotted as a function of the inflation factor and the length scale of the covariance localization under three different observation strategies in which (i) all model variables, (ii) every second variable, and (iii) every fourth variable were sampled every day.

As expected, all three filters have smaller RMSE with denser observation networks in all tested cases. Covariance localization and inflation clearly improve the filters' performance and can prevent the filters' divergence in all three observation strategies. Overall, the minimum RMSE is comparable for all three filters, but the shape of the RMSE function varies with the observation strategies as well as with the filters. When all variables are observed, the minimum RMSE of the AEnKF and EnKF are very close, but the EnKF is more sensitive to the changes of the inflation factor and the localization length scale. Therefore, a small change in the value of the length scale of the localization factor can easily cause the divergence of the EnKF. 
If we write the term "stability" to indicate the insensitivity of the RMSE function to the changes in the inflation factor and the localization length scale, the hybrid scheme is the most stable for all observation networks. But it has the largest RMSE minimum with dense observation network. This may partially be due to the fact that relaxing the EnKF covariance matrix to an invariant $\mathbf{B}$ introduces unnecessary features limiting the hybrid filter's performances. In contrast, the adaptive approach filters out these features and yields RMSE close to the minimum value for any choice of inflation and localization. Although the RMSE functions for all filters are less stable when only half of the variables are observed, the AEnKF provides the best solution. In the case with even fewer observations, however, the residuals contain less information. This badly affects the performances of the adaptive approach and limits its performance, although its minimum RMSE is still among the lowest. Less observation network also degrades the hybrid solution, but to a lesser degree than the adaptive.

To analyze the role of the newly generated member in the distribution of the EnKF ensemble, the time evolution of the first model variable $x(1, t)$ over a 21-day period (between days 80 and 100) as estimated by the EnKF and the AEnKF is shown in Fig. 2 before (forecast) and after (analysis) applying the filter correction step. Plots are shown for the case where observations of all model variables were assimilated, and a localization length scale of 10.95 and an inflation factor of 1.01 were used. In this setting, the EnKF fails to accurately recover the reference states while the AEnKF shows much better performance (Fig. 1). This provides a good test case to examine how the addition of the back-projected residuals can improve the behavior of the EnKF. In Fig. 2, the thin solid line represents the true state, the thick solid line represents the mean of the ensembles (or the filter estimates), and gray lines represent the evolution of all the ensemble members in time. The black dots in Fig. 2d point to the locations of the new members generated after every analysis step according to (10).

At day 83 both filters have a similar analysis quite close to the observation, and similar spread (Figs. 2b,d). As the system is integrated forward in time, the differences between the distribution of the true states and the ensembles of the two filters become more pronounced. At day 86, both filters fail to accurately forecast the reference solution. Beyond this date, the EnKF misses the trajectory of the reference states. Similar behavior of the EnKF is observed all over the assimilation window. The decrease in the ensemble spread of the EnKF is generally associated with a weak fit to the observations. In contrast, increased residuals allow more information to be available, and the AEnKF seems to extract it effectively. The AEnKF new members are always generated in such a way as to enhance the distribution of the ensemble around the true state, providing information about the part of the distribution that was misrepresented by the filter ensemble. With enough information from the observations, the adaptive approach clearly allows the filter to adjust its ensemble when needed, enabling the filter to accurately track the true trajectory of the system through the different model regimes as can be seen from Fig. $2 d$.

\section{c. Case with only model error}

In this section we tested the AEnKF in the presence of model errors and evaluated its performance with respect to the EnKF and the hybrid approach. To limit the impact of the sampling error, a relatively large ensemble of 300 members was used, supposedly enough to accurately represent the distribution of the filters' covariance matrices. Limitations in the filters' background covariance matrices are then assumed to be only due to model error.

To introduce model error in L96, the assimilation runs were carried out with incorrect forcing $F$ in (23). Specifically, $F$ was set to 6 in the forecast model used to update the ensemble forward in time, while the reference states were forced with $F=8$. Two different series of experiments were then performed. In the first series, the model error was accounted for in the forecast model by adding centered Gaussian noise with a standard deviation equal to 2 . This is the filter run with an imperfect model. In the second series, the model error was not accounted for in the filters, which means that the model was assumed perfect. In all experiments performed in this section, observations were sampled from all model variables and assimilated every day. Both covariance localization and inflation were also used. Because of the large size of the ensemble in these experiments, the AEnKF was more sensitive to the value of $\beta$ and the number of ensemble members to be replaced in the ensemble, and both were tested in the experiments described below. In general, a larger value of $\beta$ and more new members both increase the impact of the backprojected information from the residuals, further shifting the mean of the new ensemble toward the data.

Figure 3 shows the RMSE for filter runs using different values of inflation factors and length scales of covariance localization. Results are shown for the cases without (left bars - perfect model) and with (right barsimperfect model) accounting for the model error in the filters. From left to right, the five bars in each group indicate the RMSE value of the EnKF, AEnKF, AEnKF with $\beta=5$, AEnKF with 30 new members, and hybrid 
(a) EnKF, Forecast

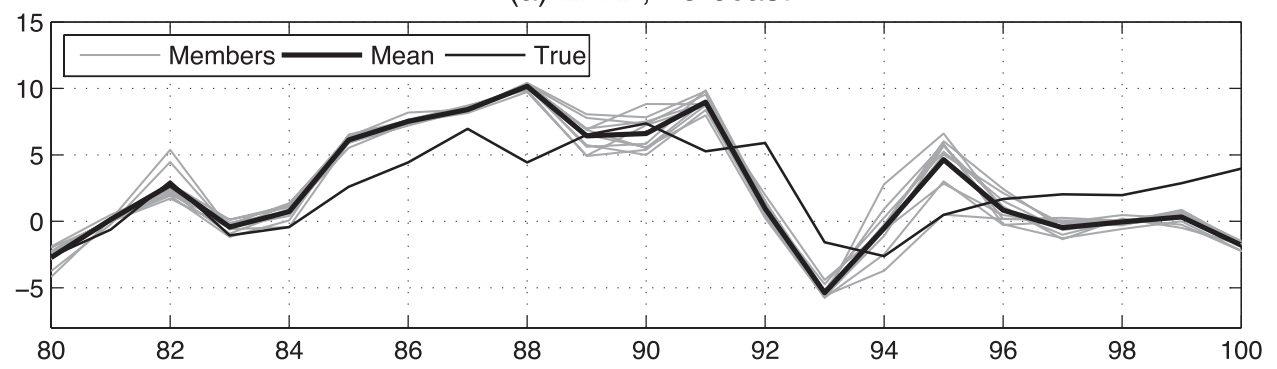

(b) EnKF, Analysis

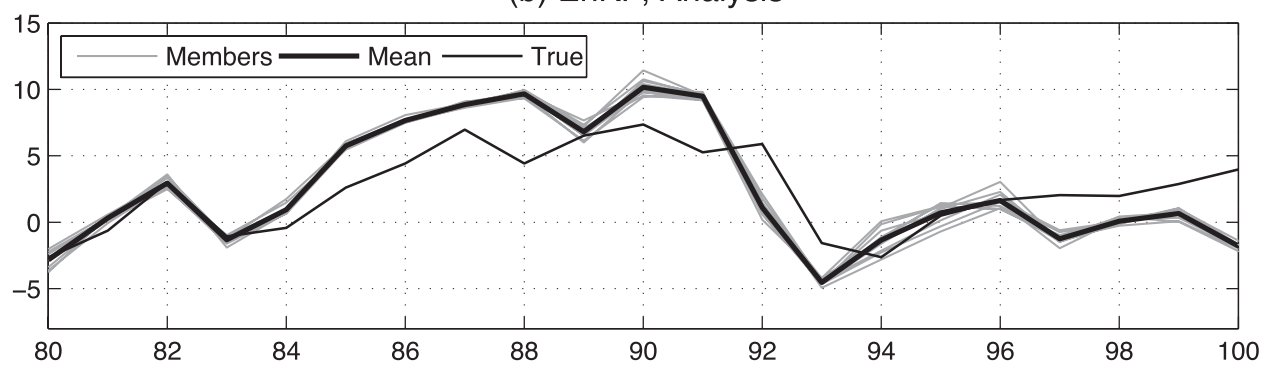

(c) AEnKF, Forecast

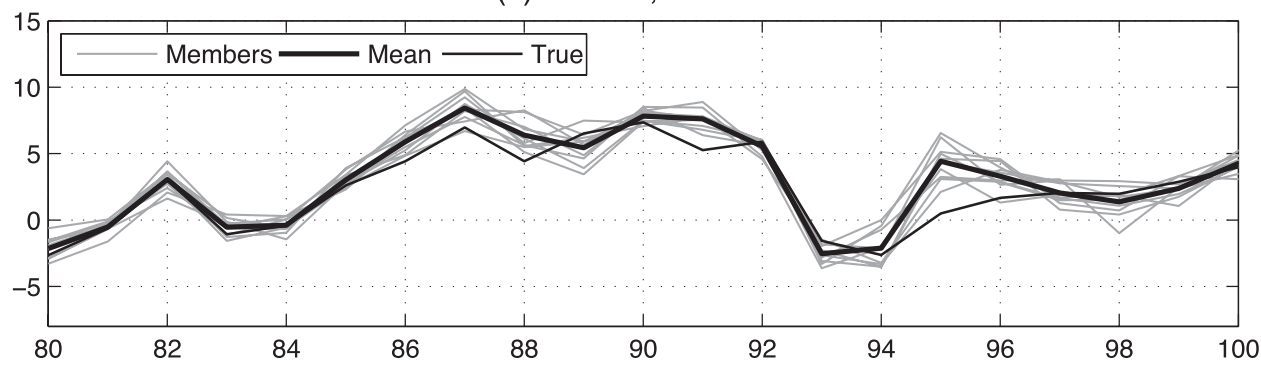

(d) AEnKF, Analysis

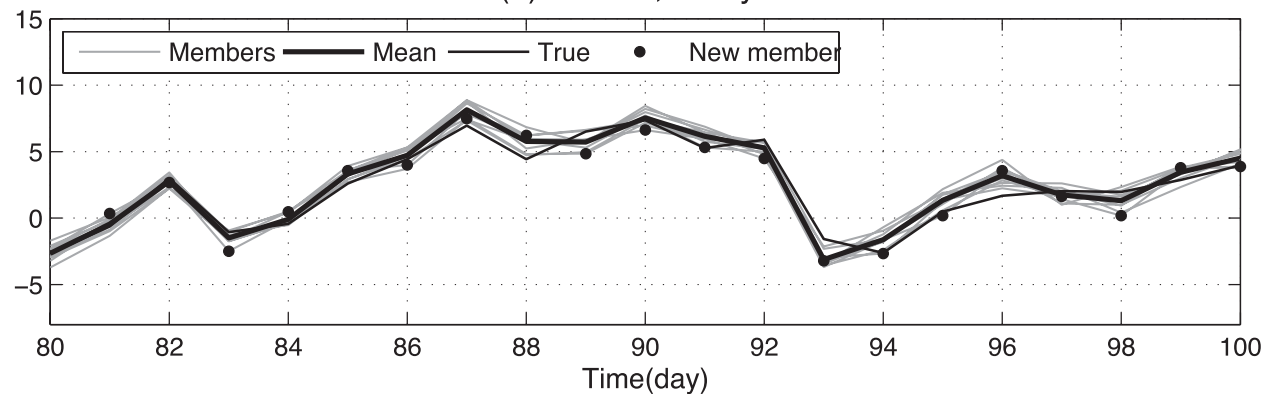

FIG. 2. Time evolution of the first model variable $x(1, t)$ between days 80 and 100 in the true state (thin black line), mean of ensemble members or filters' estimates (thick black line), and 10 ensemble members (gray lines), as it results from (a) EnKF forecast, (b) EnKF analysis, (c) AEnKF forecast, and (d) AEnKF analysis. The new member is indicated with black dots in (d). No model error is introduced in these experiments.

EnKF-3DVAR, respectively. Each bar represents the averaged error from 30 runs starting from different initial conditions. The 30 new members were randomly generated using a second-order resampling scheme as discussed in section 2 . These were added to the ensemble after removing the 30 members that contributed the least to the spread of the ensemble.
Perturbing the forecast model with random noise can clearly account for the model error (right bars). The differences in performance between the three filters are insignificant and all three were able to accurately estimate the reference states. Inflation and localization have almost the same impact on all filters and not surprisingly, the best performances were obtained with higher inflation 

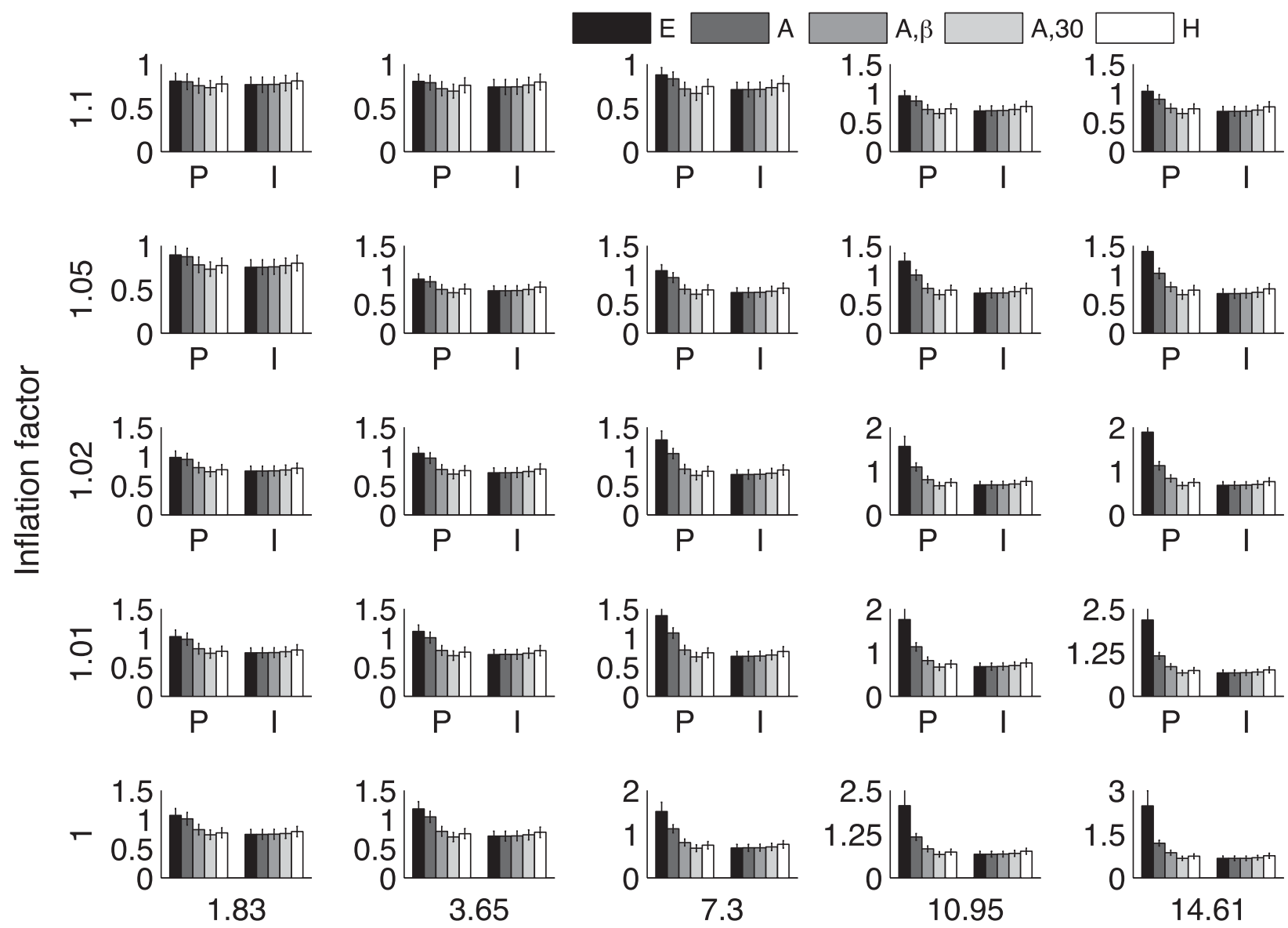

\section{Covariance Filter Length scale}

FIG. 3. RMSE and error bar as a function of (left to right) the length scale of the error covariance correlation and (bottom to top) inflation factor. The 300-ensemble EnKF, AEnKF, AEnKF with $\beta=5$, AEnKF with 30 new members, and a hybrid scheme with incorrect forcing were used to assimilate the observations sampled from all variables under both perfect model (P) and imperfect model (I) assumption in the presence of model error: (left to right) covariance filter length scale from 1.83 to 14.61. Each group has five bars. (from left to right) RMSE of EnKF, AEnKF, AEnKF with $\beta=5$, AEnKF with 30 new members, and hybrid of EnKF-3DVAR.

and stronger localization. The adaptive and the hybrid approaches have almost an insignificant impact on the EnKF. With practically no sampling error and efficient accounting for model error, the EnKF is able to accurately estimate the background covariance matrix and no extra treatments are needed.

From the left group of bars in Fig. 3, one can see that not accounting for model error can significantly degrade the performances of the EnKF. The EnKF behaves best with strong localization and inflation, and this is also true for the AEnKF and the hybrid filter. The better results obtained with the AEnKF over EnKF suggest that adding only one member can enhance the representation of an ensemble of 300 members and partially account for model error. This, however, is not enough to shift the mean closer to the observations. Increasing the weight of the new member using $\beta=5$ clearly improves the performances of the AEnKF. Note that larger values of $\beta$ might however cause the divergence of the forecast model in the AEnKF. Adding more members (e.g., 30) to the ensemble is also beneficial and further improves the performance of the AEnKF. More tuning to find the optimal parameters was not performed but is expected to lead to better results. Under this setup, the best performances were obtained with the AEnKF with 30 new members although the differences in performances were not statistically significant.

To better assess the behavior of the AEnKF, Fig. 4 plots the time evolution of the first model variable $x(1, t)$ over a 21-day period (between days 90 and 110) as it is estimated by the AEnKF with $\beta=5$ and the AEnKF with 30 new members before (forecast) and after (analysis) 
(a) AEnKF with $\beta=5$, Forecast

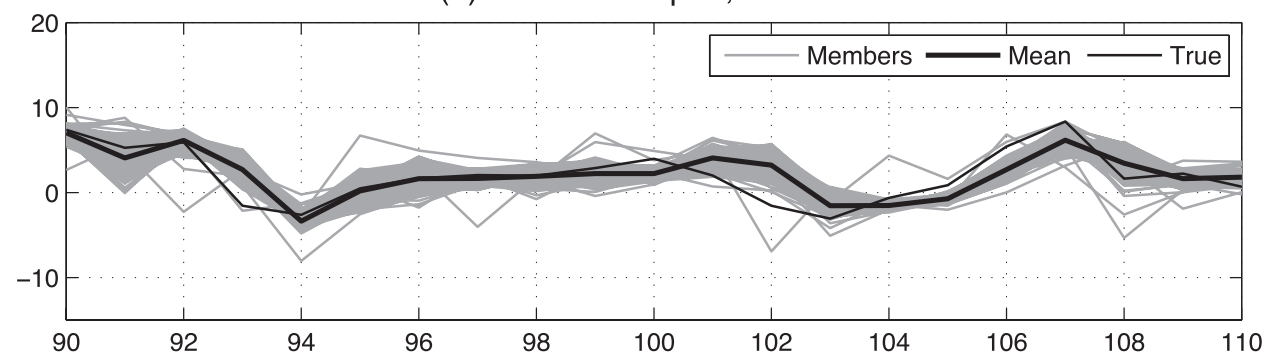

(b) AEnKF with $\beta=5$, Analysis

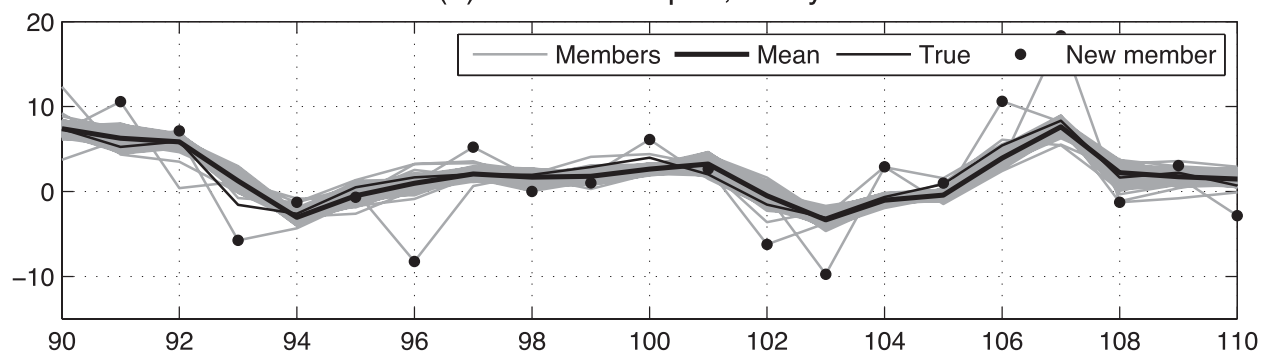

(c) AEnKF with new 30 member, Forecast

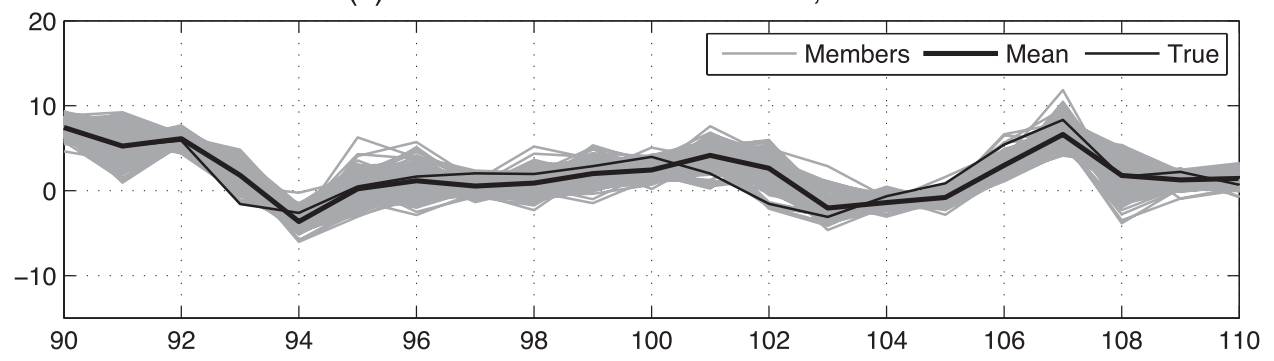

(d) AEnKF with new 30 member, Analysis

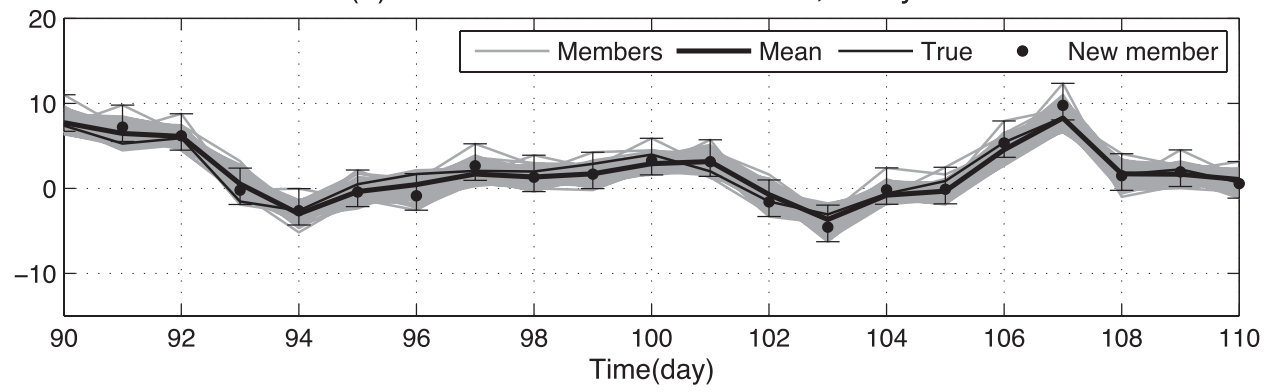

FIG. 4. Time evolution between days 90 and 110 of the true state (black thin line), mean of ensemble members (black thick line), and the 300 ensemble members (gray lines) for the first model variable $x(1, t)$ as it results from (a) AEnKF forecast with $\beta=5$, (b) AEnKF analysis with $\beta=5$, (c) AEnKF forecast with 30 new members, and (d) AEnKF analysis with 30 new members. The new member is indicated with black dots in (b) and (d), and the other 29 new members are shown with the error bars in (d).

the correction step. The inflation factor and localization length scale were set to 1.01 and 10.95 , respectively, and all variables were sampled as observations. The true state is plotted by the thin black line. The filter ensemble members are plotted in gray lines and the ensemble mean (filter estimate) is plotted in the thick black. Black dots show the locations of the newly generated members. Error bars in Fig. 4d indicate the range of the 30 new members. It is clear from Fig. $4 \mathrm{~b}$ that a large value of $\beta$ tends to increase the spread of the ensemble trying to improve the fit to the observations. Larger values of $\beta$ might further shift the position of the new member. This might, however, destroy the dynamical consistency of the newly generated member and can provoke the divergence of the forecast model. Adding 30 members clearly improves the 

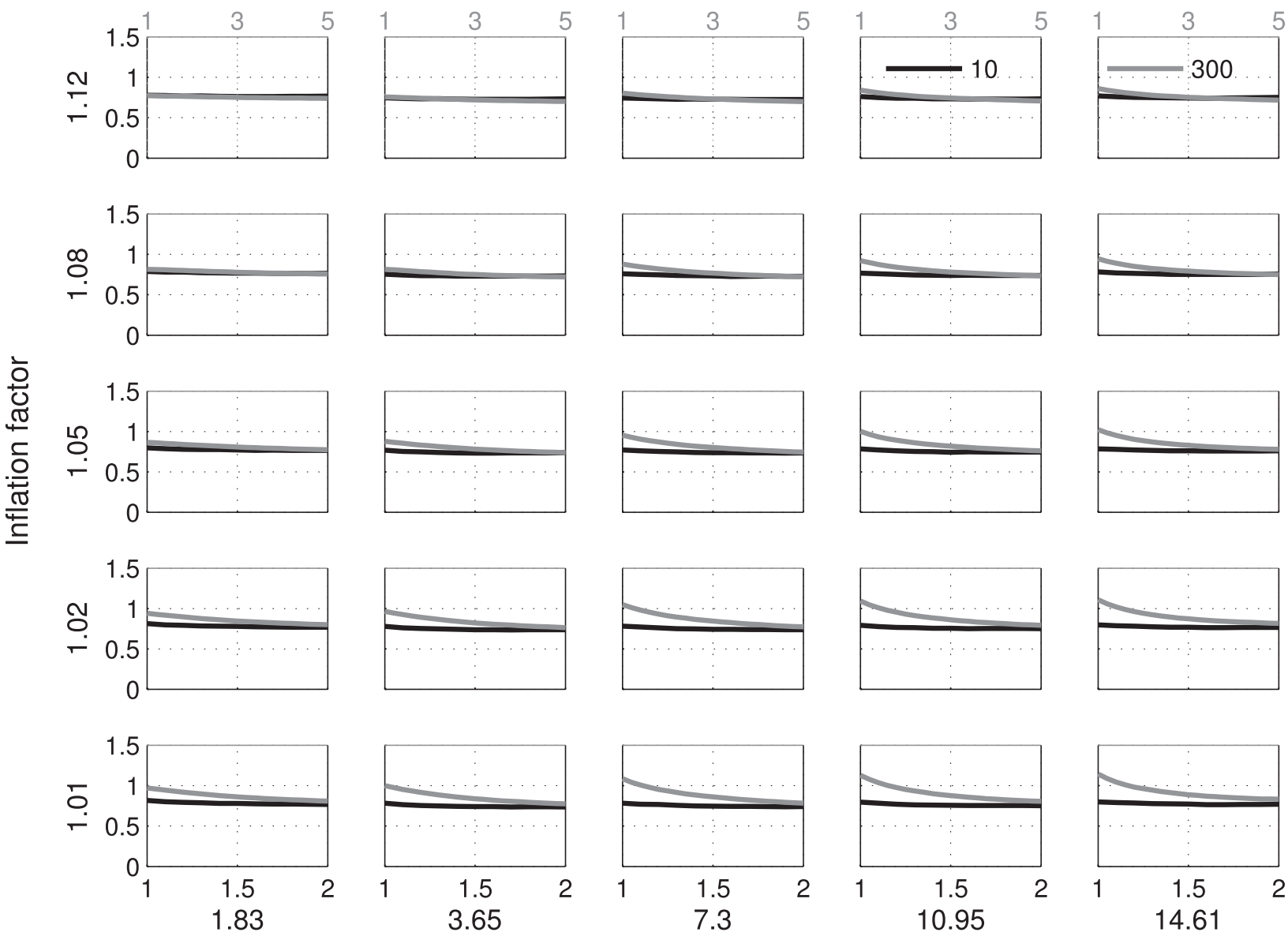

Covariance Filter Length scale

FIG. 5. RMSE of the AEnKF as a function of (left to right) the length scale of the error covariance correlation, (bottom to top) inflation factor, and $\beta$ for a filter assimilating all model variables in the presence of model error. A set of different $\beta$ values were applied to the new member for 10-member (black) and 300-member (gray) ensembles. The factor $\beta$ ranged from 1 to 2 with a 0.1 interval for the 10-member ensemble and from 1 to 5 with a 0.2 interval for the 300 -member ensemble.

spread of the ensemble, resulting in better estimates of the model variables. The new members are also shown to be more evenly spread around the filter state. The analysis step always improves the distribution of the ensemble around the true estimate. The true and estimated solutions are located within the spread of the new members, suggesting an efficient redistribution of the ensemble after every analysis step with the AEnKF.

These results suggest that the behavior of the AEnKF with large ensembles can be improved with values of $\beta$ greater than 1 . The RMSE of the AEnKF with 300 ensemble members plotted in gray in Fig. 5 seems to converge to a lower value as $\beta$ increases from 1 to 5 , although stronger localization and greater inflation reduce the effect of $\beta$. With a small ensemble of 10 members, the RMSE of the AEnKF plotted in black seems to be weakly sensitive to different values of $\beta$.
This is because the new member has a higher relative weight in an ensemble of 10 members than in an ensemble of 300 members. These results suggest that the improvement of the ensemble is mainly carried by the addition of the new direction and less by the weight of the new member. With a large ensemble, however, the error covariance is hardly changed after the addition of one new member. A large value of $\beta$ is then needed to increase the weight of the new member in the ensemble in order to improve the ensemble spread. As $\beta$ increases, the information contained in the new member is better represented in the ensemble, resulting in smaller RMSE. This is, however, only true up to a certain value because large values of $\beta$ could sometimes lead to a new member far away from the true trajectory of the system. The effect of the new member to the ensemble mean and the error covariance is further discussed in section $4 \mathrm{~d}$. 
(a) EnKF, all

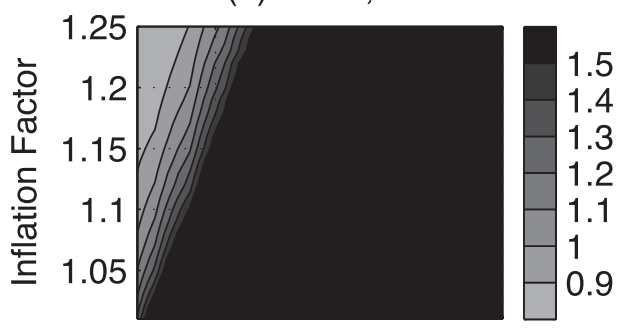

(d) Hybrid, all

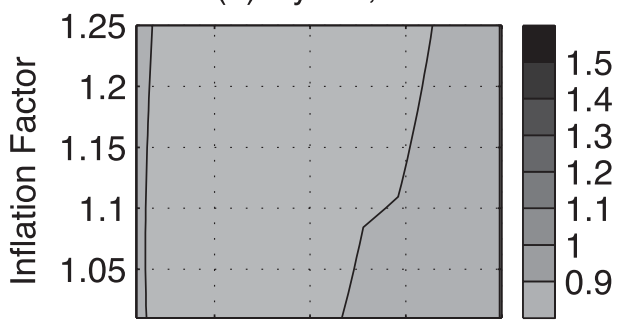

(g) AEnKF, all

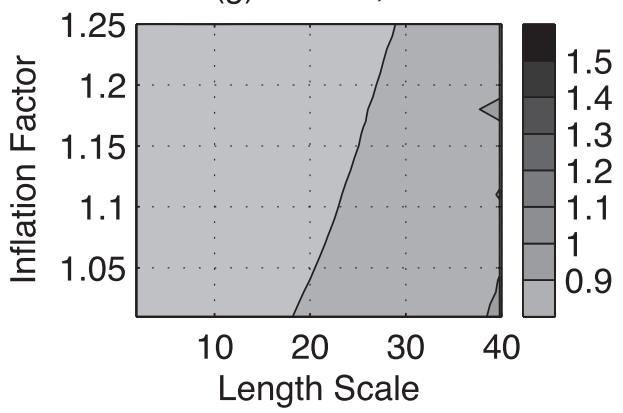

(b) EnKF, half

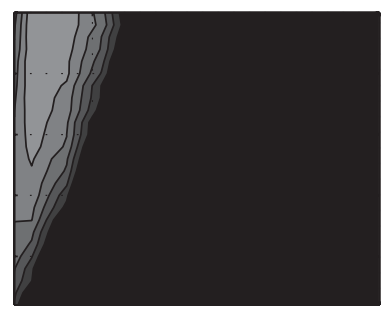

(e) Hybrid, half

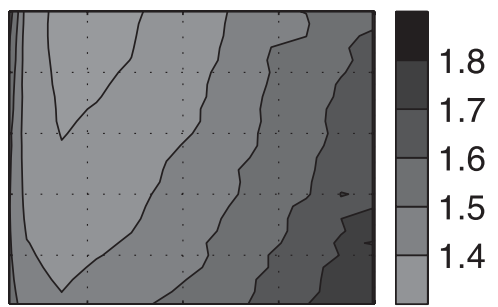

(h) AEnKF, half

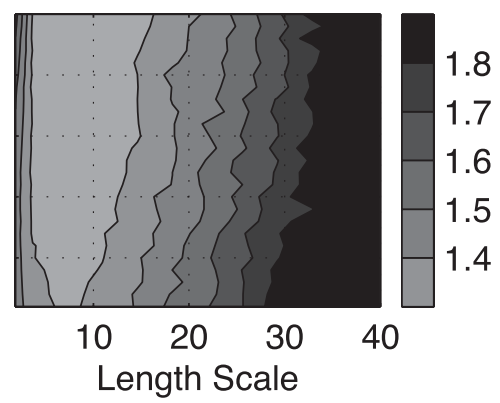

(c) EnKF, quarter

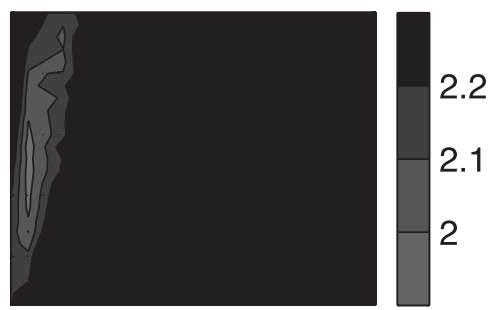

(f) Hybrid, quarter

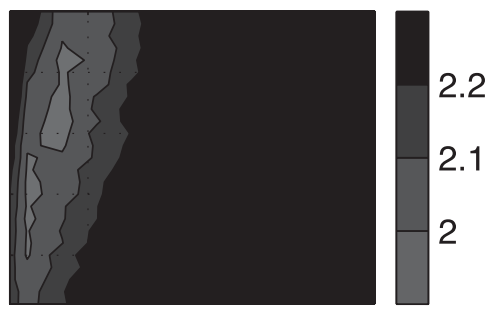

(i) AEnKF, quarter

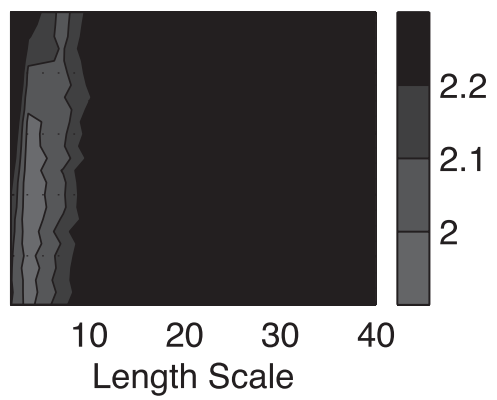

FIG. 6. As in Fig. 1, but the model error is introduced and the model is assumed perfect (model error was not accounted for in the filters) so that both sampling and model error are present.

\section{d. General case}

In the last series of experiments, the filters were tested in a more realistic case where both sampling error and model error are present in the system. The filters were implemented with an ensemble of 10 members, causing sampling error in the EnKF background covariance matrix. Model error was also included in the same way as in section 4c. The model was assumed perfect, meaning that model error was not accounted for in the filters. (Cases where the model was assumed to be in error were also run, but the differences between filters were not statistically significant, as can be seen from the right groups of bars in Fig. 10.)

The filters were implemented for three observation networks assimilating data every fourth model time step (every day). The RMSE for each run is plotted as a function of the localization length scale and the inflation factor in Fig. 6. As in the perfect model case (without model error; Fig. 1), all filters show lower RMSE with more observations. Not surprisingly, the RMSE of all filters is greater than in the cases where either only the sampling error or only the model error was included. The ranges of parameters that minimize the RMSE of all filters are also smaller, especially in the case where fewer observations were assimilated. Larger inflation and shorter localization length scales are generally needed for the filters to perform best. Overall, AEnKF and hybrid EnKF-3DVAR are comparable showing the improvement over EnKF in all three observation systems. For "quarter" sampling, the improvement is not as great as other sampling cases, which suggests the lack of new information for filters.

The rank histogram, also known as the Talagrand diagram, provides an indication of the reliability of the filter ensemble (Hamill and Snyder 2000). Ideally, if an event has a certain probability of occurring, the ensemble should suggest the same probability. This is true if the value of an observation (or of the true state if available) has an equal chance to occur in any of the possible ranks relative to the sorted ensemble (from low to high). Over many samples, this is reflected by a flat 


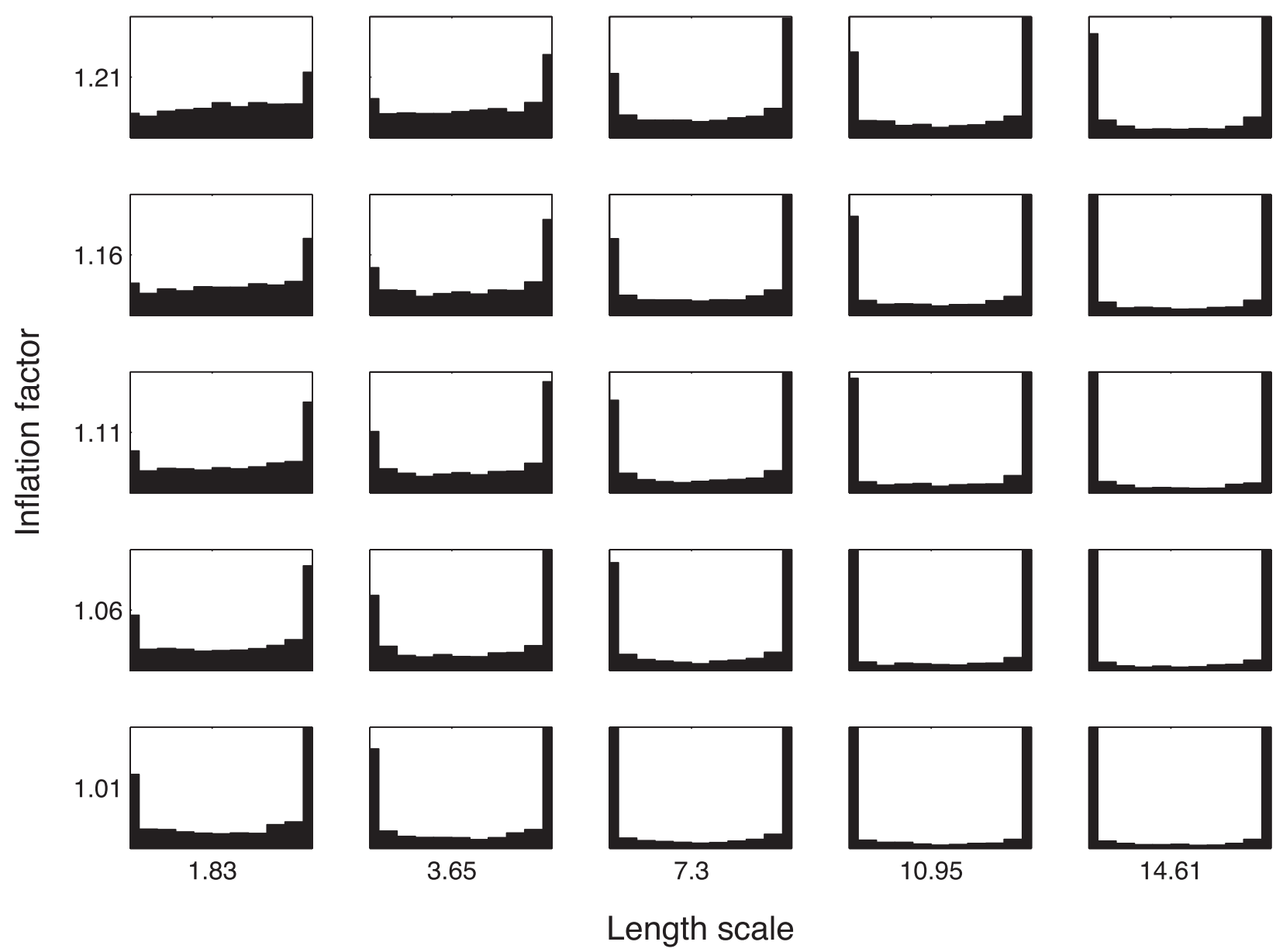

FIG. 7. Rank histograms of 10-member EnKF as a function of (left to right) the length scale of the error covariance correlation and (bottom to top) the inflation factor. Observations of all model variables were assimilated. The model error was introduced by forcing the forecast model with an incorrect value.

histogram. Nonuniformity in rank histograms might suggest potential problems in the ensemble. For example, if the ensemble spread is not sufficient or is biased, then the rank histogram will have higher values at one or both edges (i.e., $U$ shape). If the ensemble spread is overestimated, then the rank histogram has low values at the edges and is concave downward (Anderson 1996a; Hamill and Snyder 2000).

The rank histograms from the EnKF, the hybrid EnKF-3DVAR, and the AEnKF under different localization length scales and inflation factors are shown in Figs. 7-9, respectively, for the case where observations of all model variables were assimilated. Since the model used for assimilation is biased because of the incorrect forcing, all filters generally show the histograms that are more populated on the right-hand side. The extreme U shapes in the rank histogram of the EnKF correspond to the cases where the ensemble has small variance preventing the filter analysis from decreasing the RMSE.
The rank histogram becomes more uniform with more aggressive localization. Inflation seems to have a smaller impact on the filter rank histogram, although in most cases, greater inflation improves the distribution of the ensemble. The rank histograms of the hybrid EnKF3DVAR in Fig. 8 are relatively more uniform, but inclined to the right. There is, however, a trend to overpopulate the center of the histogram as the localization length scale gets short and inflation increases, typically indicating an excess of variability in the ensemble. The rank histograms of the hybrid method show a consistency with the discussion in section 3 suggesting that covariance compensation techniques, such as inflation and localization, should have less impact on the its performance. The rank histograms of the AEnKF have a slight U shape with the biggest population on the most right-hand side, and the flattest (best) histograms were obtained with strong localization and moderate inflation. They are more uniform than those of the EnKF in all tested cases. 


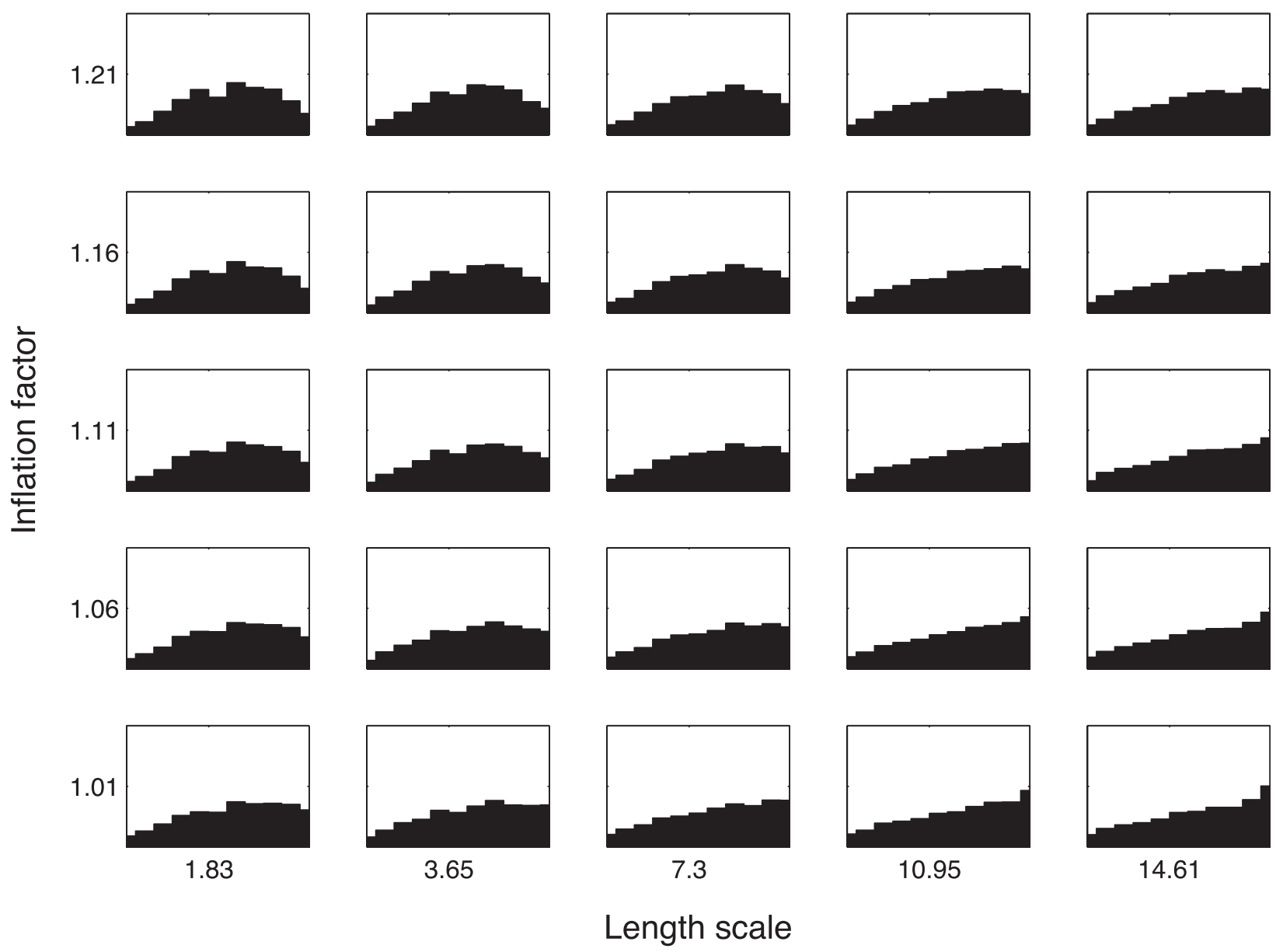

FIG. 8. As in Fig. 7, but for hybrid EnKF-3DVAR.

Figure 10 shows the time mean RMSE over all variables for EnKF, AEnKF, and hybrid EnKF-3DVAR when all variables were observed. As before, the group of bars on the left in each subplot show the RMSE when the forecast model was assumed perfect and the group of bars on the right represent cases where the model error was accounted for by adding random noise during the forecast step. The error bars indicate the averaged error from 30 runs starting from different initial conditions. As shown in Fig. 6, the EnKF is sensitive to the choice of the length scale under the perfect model assumption. EnKF diverges as the RMSE increases and the ensemble variance shrinks (not shown) when the error covariance correlation length scale is larger than 10 , particularly when the covariance is inflated by less than $10 \%$. However, the AEnKF maintains a good fit to observations, showing RMSE smaller than EnKF and comparable to hybrid 3DVAR-EnKF with most different settings.

To study modifications to the direction and the amplitude of the ensemble by the new member, we analyze the estimate of the first variable, $x(1, t)$ as it resulted from the experiment with an inflation factor $=1.01$, a localization length scale $=10.95$, and a weighting parameter $\beta=1$. Figure 11a compares the time evolution of the first variable for the true states (thick black line), the analysis ensemble mean before (thin gray line), and after adding a new member (thin black line). The difference between the ensemble mean before and after adding a new member is negligible and the two thin lines are almost indistinguishable.

A measure of improvement due to the new member in the ensemble is the normalized distance $D_{n}$ between the misfits of the true state and the ensemble mean before and after adding the new member $\mathrm{MF}_{a}=\left\|x^{t}-x^{a}\right\|$ and $\mathrm{MF}_{a, e}=\left\|x^{t}-x^{a, e}\right\|:$

$$
D_{n}=\frac{\mathrm{MF}_{a}-\mathrm{MF}_{a, e}}{\mathrm{MF}_{a}} .
$$

Positive $D_{n}$ means that the new member positively contributes to the ensemble mean. Throughout the assimilation window in Fig. $11 \mathrm{~b}, D_{n}$ is close to zero, implying 


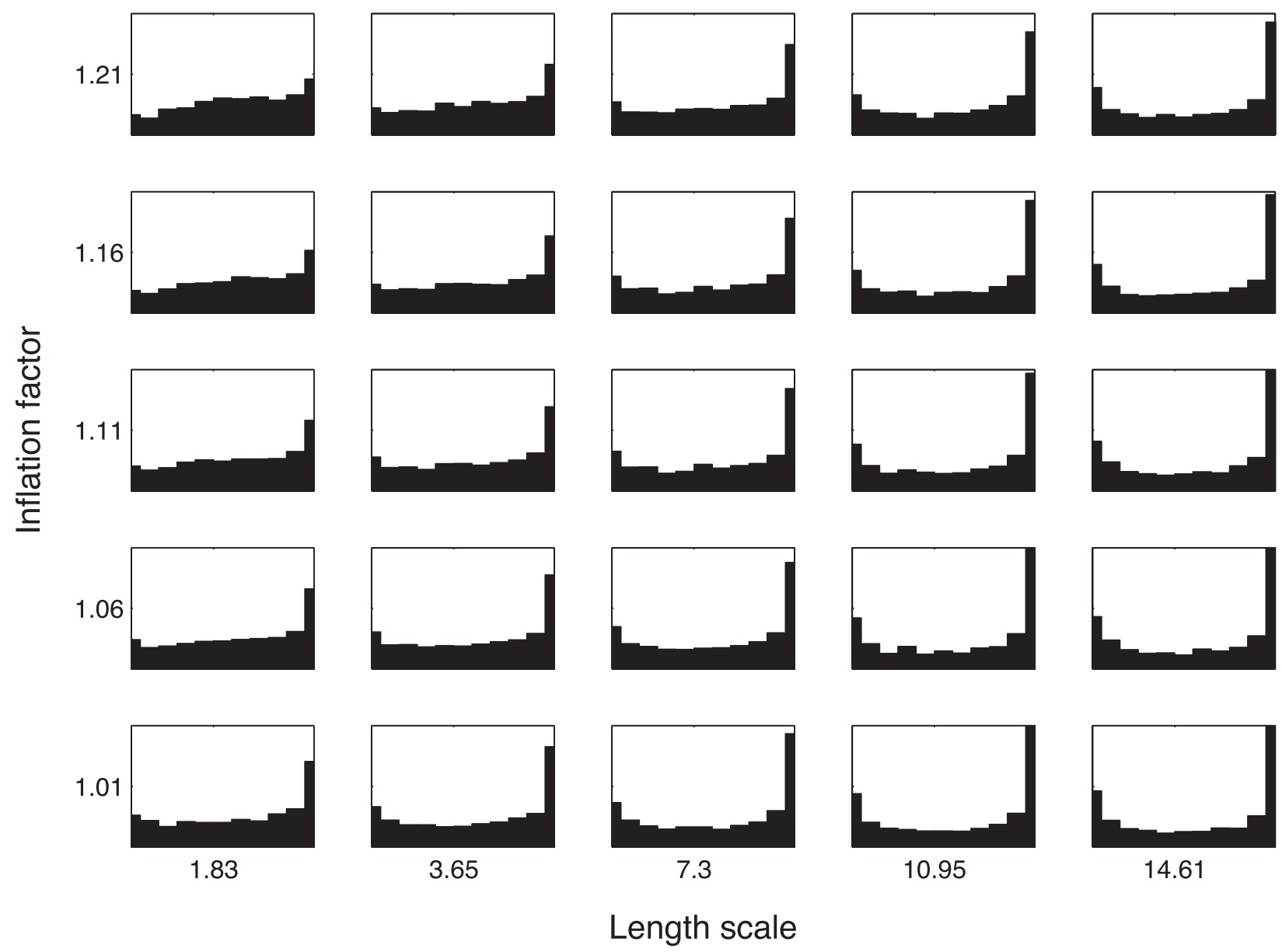

FIG. 9. As in Fig. 7, but for AEnKF.

that the correction made by the new member to the ensemble mean is negligible. This is expected because of the choice of $\beta=1$ in this experiment. One can also notice that because of observational errors, the addition of the new member does not always modify the mean in the direction of the true state.

Finally we computed the eigenvalues of the error covariance matrices to assess how the addition of the new member changes the subspaces defined by these matrices. The size of the modification is estimated from the eigenvalue spectra and/or the sum of all eigenvalues (the trace of the error covariance matrix) before and after replacing the least meaningful member with the new member to the ensemble, and it is shown in Fig. 11c. Without a new member, the trace of the error covariance starts greater than 25 and then quickly decreases to values less than 10 . With a new member, the ensemble is shown to significantly increase the eigenvalue spectra of the error covariance matrix.

If the ensemble members become similar, then the eigenvalues of the error covariance matrix may have a flat spectrum, but with small magnitude. If the adaptive process adds a new member that is significantly different, the revised eigenvalue spectrum may have an increased difference between the largest two eigenvalues, but this represents a state with more freedom. This conjecture is illustrated by the time-averaged eigenvalue spectra of the error covariance before and after adding a new member in Fig. 11d. Including the new member in the ensemble introduces a significant mode to the system. By construction, it is nearly orthogonal to the original ensemble members and so contains new information. In addition, the magnitude of the uncertainty in this new direction is large compared to the analysis ensemble before augmentation. When the ensemble includes the new member, the RMSE of the estimated states with respect to the true state is reduced by about $8.5 \%$.

\section{Discussion}

The ensemble Kalman filter (EnKF) provides a powerful and effective tool for analysis and forecasting. However, 

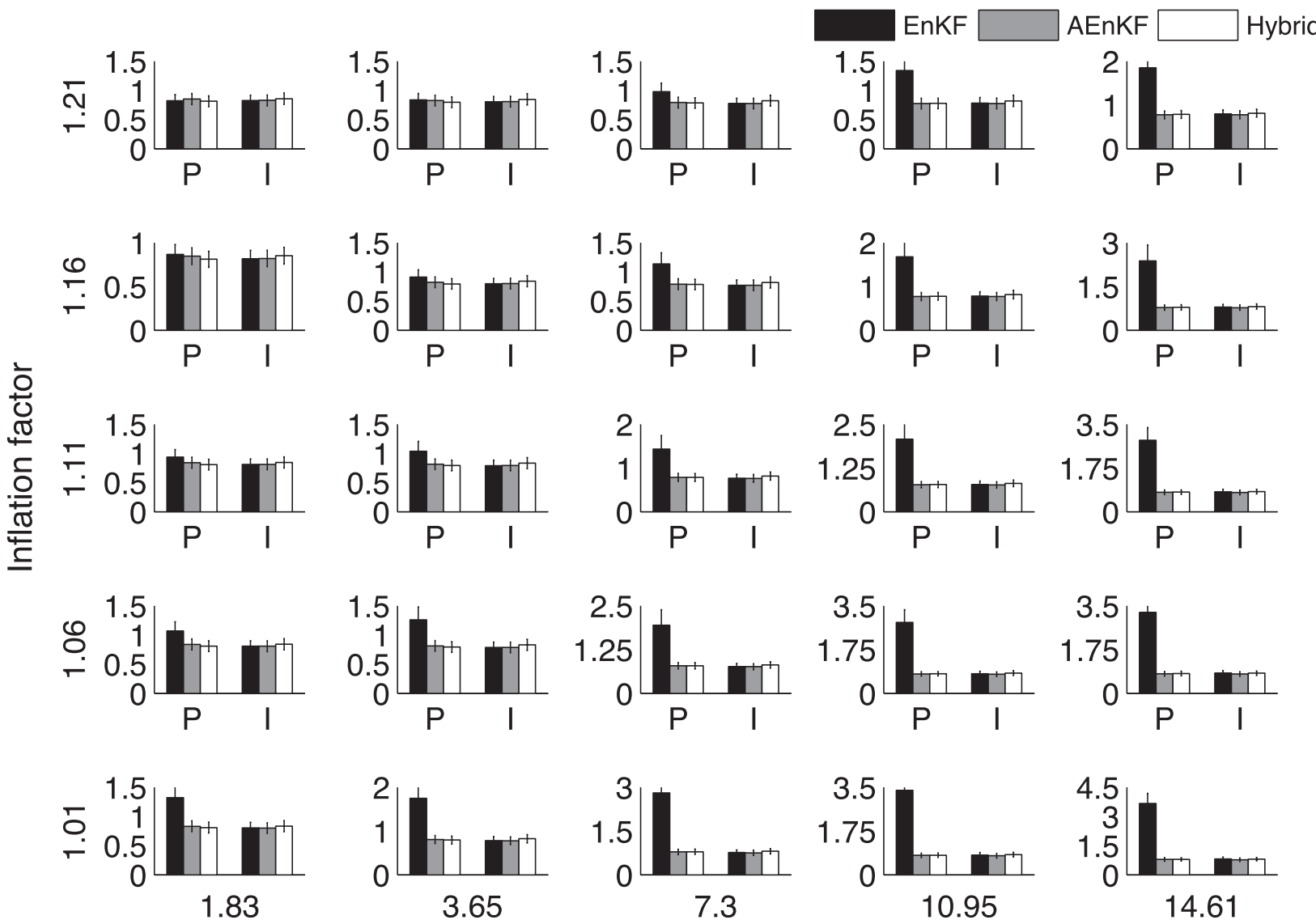

\section{Covariance Filter Length scale}

FIG. 10. RMSE averaged over all variables as they result from the EnKF, AEnKF, and hybrid EnKF-3DVAR with 10 ensemble members and with model error: (left to right) the length scale of the error covariance correlation and (bottom to top) the inflation factor. Left bars show RMSE values when model error was accounted for and right bars when not.

accounting for small ensemble size and model deficiencies remains a significant issue in ensemble data assimilation systems. These are important factors in determining the accuracy of the background covariance. Moreover, the model dynamics and the action of the filter can decrease the effective dimension of the ensemble with time. If the number of independent components (rank) of the ensemble becomes too small, the filter behavior can be seriously degraded. In addition, for realistic ocean and atmospheric data assimilation problems, model errors typically have large dimension, but ensembles large enough to account for model errors have prohibitive computational cost to integrate the ensemble members forward in time with the model. To overcome these limitations, we proposed a new adaptive approach to enrich the ensemble of the EnKF without increasing its size. The main idea is to use a part of the analysis step to estimate a new ensemble member in addition to adjusting the existing ensemble elements. The residuals above the observational noise level contain information about the structure missing from the background covariance that prevented the filter from fitting the observations. The new member is estimated by weighted back projection of the analysis residuals to the state space using a covariance matrix representing the errors in the ensemble. To maintain ensemble size, the least independent member of the ensemble is replaced by the new member. The covariance matrix of the error in the ensemble, $\mathbf{P}^{r}$, can be estimated by inflation, localization, or a direct guess of a covariance $\mathbf{B}$ as in the hybrid method. The mathematics of the method suggest a possible trade-off between using part of $\mathbf{P}^{r}$ to update the ensemble and part of it to estimate the new element. In the examples above, the weighting of the back-projected residuals in state space was performed using a stationary covariance matrix $\mathbf{B}$ as in an optimal interpolation $(\mathrm{OI})$ scheme. This way of targeting specific directions of $\mathbf{B}$ is the main difference between the hybrid EnKF-3DVAR 
(a) Time series of the first variable

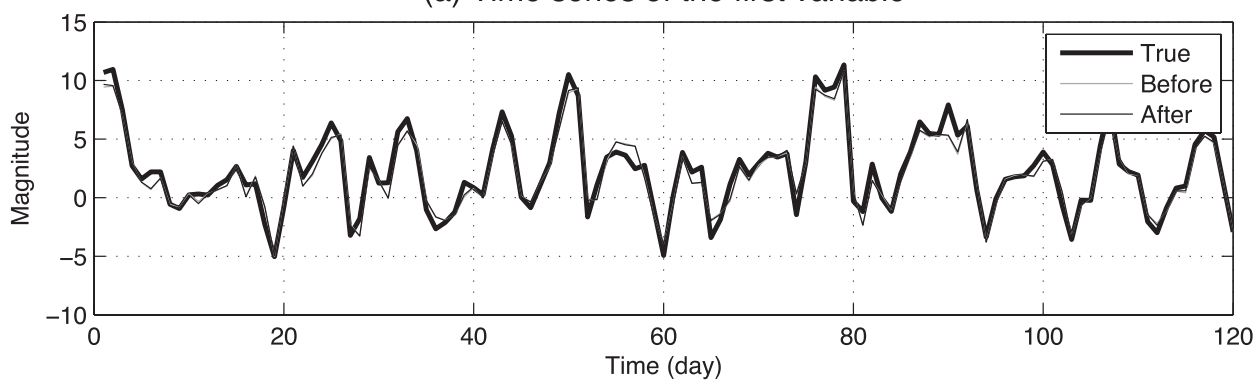

(b) Time series of the normalized distance

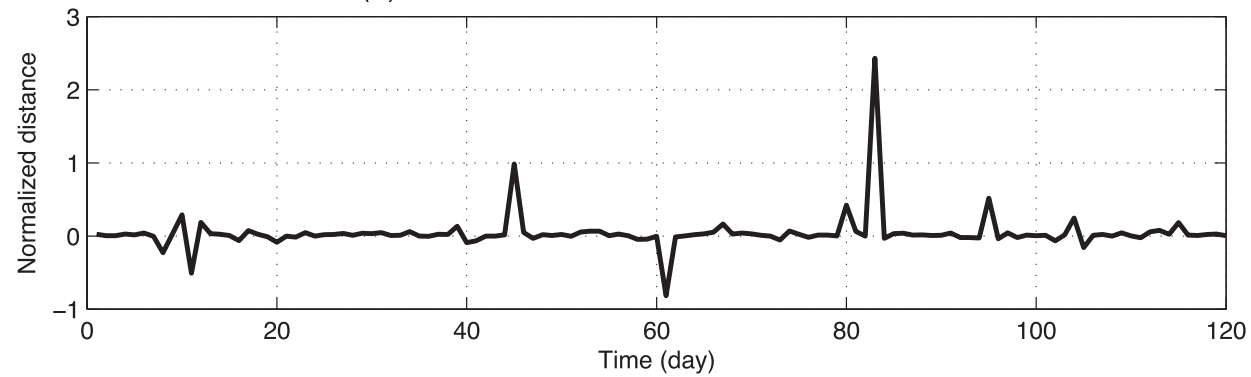

(c) Sum of all eigenvalues

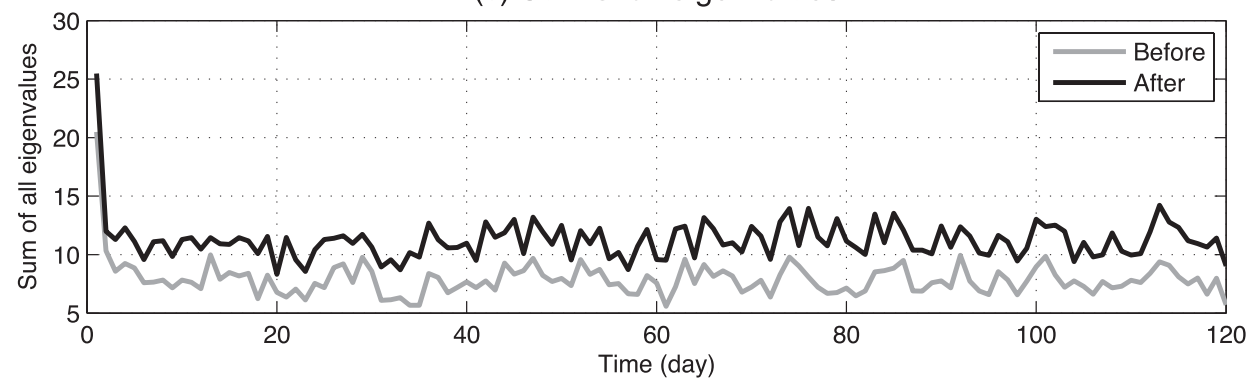

(d) Eigenvalues spectra

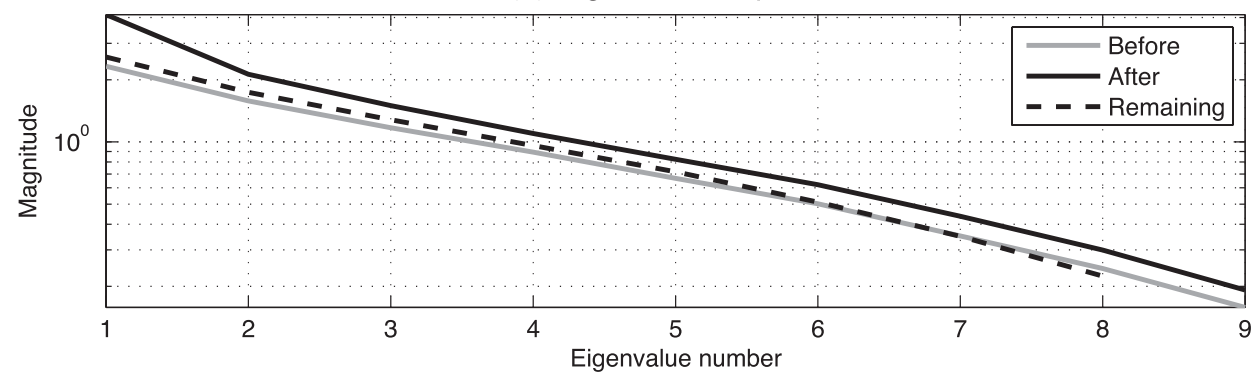

FIG. 11. (a) Time series of the first variable from the true states (thick black), the analysis before (thin gray), and after (thin black) augmenting the new member and (b) the normalized distance $D_{n}$ done by the new member. (c) Sum of all eigenvalues of the error covariance before (gray) and after (black) augmenting the new member and (d) their time-averaged normalized eigenvalue spectra with that of the error covariance excluding the new member (black dashed).

approach and the adaptive approach. The new adaptive approach can be easily implemented in any existing EnKF-based assimilation system. The selection of the new ensemble members is performed independently from the analysis step of the EnKF, and therefore no changes are needed in the EnKF algorithm.
The new adaptive EnKF, called AEnKF, was tested with the Lorenz-96 model and its performances were compared with the EnKF and hybrid EnKF-3DVAR in the presence of either sampling error, model error, or both with the background error covariance localization and the inflation factor implemented for the ensemble 
update. Preliminary numerical experiments demonstrated the utility of the adaptive scheme and the improved behavior of the traditional EnKF.

When only the sampling error was considered, the AEnKF was found to be less sensitive to the choice of the inflation factor and the length scale of the localization than the EnKF. If all variables were observed, the AEnKF is almost insensitive to these parameters, which means the RMSE does not change with any choice of the inflation factor and localization length scale. When half of the model variables were observed, the OI scheme efficiently spreads the residual information to nonobserved variables in AEnKF. As a result, the AEnKF showed better performance than hybrid EnKF-3DVAR, providing the smallest RMSE. It is also shown in the experiments that the newly generated member from the residuals could change the spread of the ensemble. As expected, the AEnKF behaved best with the densest observational coverage, as this provides more information to the adaptive scheme.

With good ensemble sampling but unaccounted-for model error, the AEnKF again successfully assimilated the observations. Experiments performed with covariance limitations only due to model error suggested that the EnKF performance might be seriously degraded if the model error was not accounted for in the forecast model used by the filter to integrate the ensemble forward in time. The EnKF was further quite sensitive to the choice of covariance inflation and localization. With an appropriate choice of parameters and number of members to be added to the ensemble, experiments suggest that the adaptive approach can significantly improve the performance of the EnKF decreasing the RMSE for a wide range of inflation and localizations values.

When both ensemble sampling and model error are present, all filters showed generally increased RMSE compared to cases with either sampling error or model error only. The EnKF again had reduced performance when the length scale was longer than 10 and when the inflation factor smaller than 1.05. The AEnKF was able to fit the observations when all and half the model variables were observed, but its performance is limited when only a quarter of the state variables were observed. AEnKF and hybrid 3DVAR-EnKF are comparable for all observation networks.

If the sampling error and the model error are both negligible, EnKF and AEnKF are similar. The newly generated member should be close to the mean of the ensemble, and the addition of this member will not introduce new information to the ensemble.

The simplified implementation of the AEnKF to the Lorenz-96 model and the validation were a necessary step before trying realistic applications, and AEnKF provided encouraging results. Many other aspects of the performance of the AEnKF remain to be explored. For example, the performance of the adaptive scheme is determined by the choice of $\mathbf{P}^{\mathrm{r} 2}$, so different forms should be tested. The option of augmenting the ensemble by more than one member at a time in order to account for uncertainty in cases with sparse observations or to increase the contribution of the adaptive members to the ensemble, which would shift the mean further toward the observations, should also be tested. Similarly, if the adjoint of the model is available, the residuals can be back propagated to earlier steps. This enables the updating of the ensemble before the problems are large enough to emerge above the observation noise, and it increases the influence of the dynamics as compared to the arbitrary matrix $\mathbf{P}^{\mathrm{r} 2}$. This is an alternative way to hybridize 4DVAR with the EnKF, and a study of these extensions to the basic scheme is underway.

Acknowledgments. Ibrahim Hoteit and Bruce Cornuelle were supported by ONR Grant N00014-08-1-0554. The authors are very grateful for Dr. Jeffrey Whitaker for valuable help and support during part of this work. The authors would like also to thank two anonymous reviewers for valuable comments and suggestions.

\section{REFERENCES}

Anderson, J. L., 1996a: A method for producing and evaluating probabilistic forecasts from ensemble model integrations. J. Climate, 9, 1518-1530.

_ 1996b: Selection of initial conditions for ensemble forecasts in a simple perfect model framework. J. Atmos. Sci., 53, 22-36.

—_, and S. L. Anderson, 1999: A Monte Carlo implementation of the nonlinear filtering problem to produce ensemble assimilations and forecasts. Mon. Wea. Rev., 127, 2741-2758.

Buehner, M., 2005: Ensemble-derived stationary and flowdependent background error co-variances: Evaluation in a quasi-operational NWP setting. Quart. J. Roy. Meteor. Soc., 131, 1013-1043.

Burgers, G., P. V. Leeuwen, and G. Evensen, 1998: Analysis scheme in the ensemble Kalman filter. Mon. Wea. Rev., 126, 1719-1724.

Evensen, G., 1994: Sequential data assimilation with a nonlinear quasi-geostrophic model using Monte Carlo methods to forecast error statistics. J. Geophys. Res., 99, 10 143-10 162.

_ 2003: The ensemble Kalman filter: Theoretical formulation and practical implementation. Ocean Dyn., 53, 343-367.

Gaspari, G., and S. E. Cohn, 1999: Construction of correlation functions in two and three dimensions. Quart. J. Roy. Meteor. Soc., 125, 723-757.

Ghil, M., and P. Malanotte-Rizzoli, 1991: Data assimilation in meteorology and oceanography. Advances in Geophysics, Vol. 33, Academic Press, 141-266.

Hamill, T., and C. Snyder, 2000: A hybrid ensemble Kalman filter3D variational analysis scheme. Mon. Wea. Rev., 128, 29052919. 
- and J. S. Whitaker, 2005: Accounting for error due to unresolved scales in ensemble data assimilation: A comparison of different approaches. Mon. Wea. Rev., 133, 3132-3147.

- - - , and C. Snyder, 2001: Distance-dependent filtering of background error covariance estimates in an ensemble Kalman filter. Mon. Wea. Rev., 129, 2776-2790.

Hoteit, I., and D.-T. Pham, 2004: An adaptively reduced-order extended Kalman filter for data assimilation in the tropical Pacific. J. Mar. Syst., 45, 173-188.

,$- \ldots$, and J. Blum, 2001: A semi-evolutive partially local filer for data assimilation. Mar. Pollut. Bull., 43, 164-174.

$\ldots, \ldots$, and $\_$2002: A simplified reduced-order Kalman filtering and application to altimetric data assimilation in the tropical Pacific. J. Mar. Syst., 36, 101-127.

— , G. Triantafyllou, and G. Korres, 2007: Using low-rank ensemble Kalman filters for data assimilation with high dimensional imperfect models. J. Numer. Anal. Ind. Appl. Math., 2, 67-78.

Houtekamer, P. L., and L. Mitchell, 1998: Data assimilation using an ensemble Kalman filter technique. Mon. Wea. Rev., 126, 796-811.

Kalman, R. E., 1960: A new approach to linear filtering and prediction problems. J. Basic Eng. Trans. ASME, 82, 35-45.
Le Dimet, F., and O. Talagrand, 1986: Variational algorithms for analysis and assimilation of meteorological observations: Theoretical aspects. Tellus, 38, 97-110.

Lorenc, A. C., 1986: Analysis methods for numerical weather prediction. Quart. J. Roy. Meteor. Soc., 112, 1177-1194.

_ 2003: The potential of the ensemble Kalman later for NWP-A comparison with 4D-Var. Quart. J. Roy. Meteor. Soc., 129, 3183-3203.

Lorenz, E., and K. Emanuel, 1998: Optimal sites for supplementary weather observations: Simulation with a small model. J. Atmos. Sci., 55, 399-414.

Mitchell, H. L., P. L. Houtekamer, and G. Pelerin, 2002: Ensemble size, balance, and model-error representation in an ensemble Kalman filter. Mon. Wea. Rev., 130, 2791-2808.

Pham, D., 2001: Stochastic methods for sequential data assimilation in strongly nonlinear systems. Mon. Wea. Rev., 129, 1194-1207.

Todling, R., 1999: Estimation theory and foundations of atmospheric data assimilation. DAO Office Note 1999-01, 187 pp.

Wang, X., C. Snyder, and T. Hamill, 2007: On the theoretical equivalence of differently proposed ensemble-3DVAR hybrid analysis schemes. Mon. Wea. Rev., 135, 222-227.

Whitaker, J. S., and T. Hamill, 2002: Ensemble data assimilation without perturbed observations. Mon. Wea. Rev., 130, 1913-1924. 